\title{
New Bounds for Energy Complexity of Boolean Functions*
}

\author{
Krishnamoorthy Dinesh $^{\dagger} \quad$ Samir Otiv $^{\ddagger} \quad$ Jayalal Sarma $^{\dagger}$
}

\begin{abstract}
For a Boolean function $f:\{0,1\}^{n} \rightarrow\{0,1\}$ computed by a Boolean circuit $C$ over a finite basis $\mathcal{B}$, the energy complexity of $C$ (denoted by $\operatorname{EC}_{\mathcal{B}}(C)$ ) is the maximum over all inputs $\{0,1\}^{n}$ of the number gates of the circuit $C$ (excluding the inputs) that output a one. Energy Complexity of a Boolean function over a finite basis $\mathcal{B}$ denoted by $\mathrm{EC}_{\mathcal{B}}(f) \stackrel{\text { def }}{=} \min _{C} \mathrm{EC}_{\mathcal{B}}(C)$ where $C$ is a Boolean circuit over $\mathcal{B}$ computing $f$.

We study the case when $\mathcal{B}=\left\{\wedge_{2}, \vee_{2}, \neg\right\}$, the standard Boolean basis. It is known that any Boolean function can be computed by a circuit (with potentially large size) with an energy of at most $3 n(1+\epsilon(n))$ for a small $\epsilon(n)$ (which we observe is improvable to $3 n-1)$. We show several new results and connections between energy complexity and other well-studied parameters of Boolean functions.

- For all Boolean functions $f$, $\mathrm{EC}(f) \leq O\left(\mathrm{DT}(f)^{3}\right)$ where $\mathrm{DT}(f)$ is the optimal decision tree depth of $f$.

- We define a parameter positive sensitivity (denoted by psens), a quantity that is smaller than sensitivity [Cook et al., SIAM Journal of Computing, 15(1):87-97, 1986] and defined in a similar way, and show that for any Boolean circuit $C$ computing a Boolean function $f, \mathrm{EC}(C) \geq \operatorname{psens}(f) / 3$.

- For a monotone function $f$, we show that $\mathrm{EC}(f)=\Omega\left(\mathrm{KW}^{+}(f)\right)$ where $\mathrm{KW}^{+}(f)$ is the cost of monotone Karchmer-Wigderson game of $f$.

- Restricting the above notion of energy complexity to Boolean formulas, we show $\mathrm{EC}(F)=$ $\Omega(\sqrt{\mathrm{L}(F)}-\operatorname{Depth}(F))$ where $\mathrm{L}(F)$ is the size and $\operatorname{Depth}(F)$ is the depth of a formula $F$.
\end{abstract}

\section{Introduction}

For a Boolean function $f:\{0,1\}^{n} \rightarrow\{0,1\}$ computed by a Boolean circuit $C$ over a basis $\mathcal{B}$, the energy complexity of $C$ (denoted by $\operatorname{EC}_{\mathcal{B}}(C)$ ) is the maximum over all inputs $\{0,1\}^{n}$ the numbers of gates of the circuit $C$ (excluding the inputs) that outputs a one. The energy complexity of a Boolean function over a basis $\mathcal{B}$ denoted by $\mathrm{EC}_{\mathcal{B}}(f) \stackrel{\text { def }}{=} \min _{C} \mathrm{EC}_{\mathcal{B}}(C)$ where $C$ is a Boolean circuit over $\mathcal{B}$ computing $f$. A particularly interesting case of this measure of Boolean function, is when the individual gates allowed in the basis $\mathcal{B}$ are threshold gates (with arbitrary weights allowed). In this case, the term energy in the above model captures the number of neurons firing in the

\footnotetext{
*Preliminary version appeared in 24th International Computing and Combinatorics Conference (COCOON 2018).

$\dagger$ Indian Institute of Technology Madras, Chennai, India. Email: \{kdinesh, jayalal\}@cse.iitm.ac.in

${ }^{\ddagger}$ Maximl Labs, India. Part of the work was done while this author was a student at the Indian Institute of Technology Madras. Email: samir.otiv@maximl.com
} 
cortex of the human brain (see [UDM06] and the references therein). This motivated the study of upper and lower bounds [UDM06] on various parameters of energy efficient circuits - in particular the question of designing threshold circuits which are efficient in terms of energy as well as size computing various Boolean functions.

Indeed, irrespective of the recently discovered motivation mentioned above, the notion of energy complexity of Boolean functions, has been studied much before. Historically, the measure of energy complexity of Boolean functions ${ }^{1}$ was first studied by Vaintsvaig [Vai62] (under the name "power of a circuit"). Initial research was aimed at understanding the maximum energy needed to compute any $n$ bit Boolean function for a finite basis $\mathcal{B}$ (denoted by $\mathrm{EC}_{\mathcal{B}}(n)$ ). Towards this end, Vaintsvaig [Vai62] showed that for any finite basis $\mathcal{B}$, the value of $\mathrm{EC}_{\mathcal{B}}(n)$ is asymptotically between $n$ and $\frac{2^{n}}{n}$. Refining this result further, Kasim-zade [Kas92] gave a complete characterization by showing the following remarkable trichotomy: for any finite complete basis $\mathcal{B}$, either $\mathrm{EC}_{\mathcal{B}}(n)=\Theta\left(2^{n} / n\right)$ or $\Omega\left(2^{n / 2}\right) \leq \mathrm{EC}_{\mathcal{B}}(n) \leq O\left(\sqrt{n} 2^{n / 2}\right)$ or $\Omega(n) \leq \mathrm{EC}_{\mathcal{B}}(n) \leq O\left(n^{2}\right)$.

An intriguing question about the above trichotomy is where exactly does the standard Boolean basis $\mathcal{B}=\left\{\wedge_{2}, \vee_{2}, \neg\right\}$ fits in. By an explicit Boolean circuit construction, Kasim-zade [Kas92] showed that $\operatorname{EC}_{\mathcal{B}}(n) \leq O\left(n^{2}\right)$. Recently, Lozhkin and Shupletsov [LS15] states (without proof) that the Boolean circuit construction by Kasim-zade [Kas92] over the complete Boolean basis is of energy $4 n$, thus deriving that $\mathrm{EC}_{\mathcal{B}}(n) \leq 4 n$. Lozhkin and Shupletsov improves it to $3 n(1+\epsilon(n))$ by constructing a Boolean circuit of size $\frac{2^{n}}{n}(1+\epsilon(n))$ for an $\epsilon(n)$ tending to 0 for large $n$. We observe that this bounds can be further improved to be at most $3 n-1$ while the size is $2^{O(n)}$ by carefully following the construction in [LS15] (Proposition 2.2).

As mentioned in the beginning, in a more recent work, for the case when the basis is threshold gates $^{2}$, Uchizawa et al. [UDM06] initiated the study of energy complexity for threshold circuits. More precisely, they defined the energy complexity of threshold circuits and gave some sufficient conditions for certain functions to be computed by small energy threshold circuits. In a sequence of works, Uchizawa et al. [UNT10, UTN11] related energy complexity of Boolean functions under the threshold basis to the other well-studied parameters like circuit size and depth for interesting classes of Boolean functions. In a culminating result, Uchizawa and Takimoto [UT08] showed that constant depth thresholds circuits of unbounded weights with the energy restricted to $n^{o(1)}$ needs exponential size to compute the Boolean inner product function ${ }^{3}$. This is also important in the context of circuit lower bounds, where it is an important open question to prove exponential lower bounds against constant depth threshold circuits in general (without the energy constraints) for explicit functions.

Our Results: Returning to the context of standard Boolean basis $\mathcal{B}=\left\{\wedge_{2}, \vee_{2}, \neg\right\}$, we show several new results and connections between energy complexity and other Boolean function parameters. Since we are interested only in the standard Boolean basis $\mathcal{B}$, we use $\mathrm{EC}(f)$ to denote $\mathrm{EC}_{\mathcal{B}}(f)$.

Upper bounds for Energy Complexity: As our first and main contribution, we show new bounds on energy complexity of Boolean functions by two other parameters of functions, one in terms of

\footnotetext{
${ }^{1} \mathrm{~A}$ related notion has been studied in [Kis82] where the energy is the number times the gates in a circuit switches its value. Recent studies [ABN $\left.{ }^{+} 14, \mathrm{BNPS15}\right]$ looks at the energy of a circuit as a function of the voltage applied to the gates thereby allowing some gates to fail. We remark that the notion of energy of Boolean circuits studied in this paper is very different from those studied in the works mentioned.

${ }^{2}$ With values of the weights and threshold being arbitrary rational numbers, notice that this basis is no longer finite and hence the bounds and the related trichotomy are not applicable.

${ }^{3} \mathrm{IP}(x, y)=\sum_{i} x_{i} y_{i} \bmod 2$
} 
an upper bound and the other in terms of a lower bound.

For a function $f:\{0,1\}^{n} \rightarrow\{0,1\}$, let DT $(f)$ denote the decision tree complexity of the Boolean function - the smallest depth of any decision tree computing the function $f$. We state our main result:

Theorem 1.1 (Main). For any Boolean function $f, \mathrm{EC}(f) \leq O\left(\mathrm{DT}(f)^{3}\right)$.

We remark that the size of the Boolean circuit constructed above is exponential in DT $(f)$. There are several Boolean functions for which the decision trees are very shallow - a demonstrative example is the tree function (see Section 2 for a definition) computable by a decision tree of depth $\log n$. Our result implies that there is a Boolean circuit computing this function of energy $O\left(\log ^{3} n\right)$.

In terms of the energy of the circuit, this improves the bounds of Lozhkin and Shupletsov [LS15] when $\mathrm{DT}(f)$ is strictly smaller than $\sqrt[3]{n}$.

On a related note, Uchizawa et al. [UDM06], as a part of their main proof, showed a similar result for threshold decision trees which are decision trees where each internal node can query an arbitrary weighted threshold function on input variables. Let $\mathrm{DT}_{t h}(f)$ denotes the depth of the smallest depth threshold decision tree computing $f$. For a basis $\mathcal{T}$ consisting of arbitrary threshold functions, their results implies that $\mathrm{EC}_{\mathcal{T}}(f) \leq 1+\mathrm{DT}_{t h}(f)$ (see Proposition 2.5 for details). Since their construction produces a weighted threshold circuit, it does not directly give us a low energy Boolean circuit even for Boolean decision trees.

Update after our work [DOS18] in connection with Theorem 1.1: Recently (after the conference version of our work [DOS18] was published), Sun et al. [SSWX19] improved the upper bound for $\mathrm{EC}(f)$ in Theorem 1.1 from $O\left(\mathrm{DT}(f)^{3}\right)$ to $O\left(\mathrm{DT}(f)^{2}\right)$. In addition, they also showed that $\mathrm{EC}(f)=\Omega(\sqrt{\mathrm{DT}(f)})$ thereby showing that the two parameters are polynomially related. ${ }^{4}$ While this improves our main result of the conference version of the paper [DOS18] in terms of the relationship between energy complexity and decision tree complexity of a Boolean function, in terms of the lower bound that we can obtain for energy complexity, the improvement really depends on the function family considered. We demonstrate this in a comparison between the lower bound methods in Section 6.

Lower Bounds for Energy Complexity : To obtain lower bounds on energy, we define a new parameter called the positive sensitivity (which is at most the sensitivity of the Boolean function [CDR86]). Let $[n] \stackrel{\text { def }}{=}\{1, \ldots, n\}$. For a function $f:\{0,1\}^{n} \rightarrow\{0,1\}$ and an input $a \in\{0,1\}^{n}$, we define the positive sensitivity of $f$ on $a$ (denoted by psens $(f, a)$ ) as the number of indices $i \in[n]$ such that $a_{i}=1$ and flipping the bit $a_{i}$ causes the function to change its value. We define psens $(f)$ to be $\max _{a \in\{0,1\}^{n}} \operatorname{psens}(f, a)$. Using this parameter, we show the following.

Theorem 1.2. For any Boolean function $f:\{0,1\}^{n} \rightarrow\{0,1\}$ computed by a Boolean circuit $C, E C(C) \geq$ $\operatorname{psens}(f) / 3$.

The main tool in proving the above results is the notion of continuous positive paths which are paths in a Boolean circuit where all the gates in the path evaluate to 1 . Using the same tool, we show that the monotone Karchmer-Wigderson games can be solved by exchanging at most $\mathrm{EC}(C) \log c$ where $C$ is a Boolean circuit with fan-in at most $c$ (see Lemma 4.2 for more details). This implies the following energy lower bound for computing monotone functions.

\footnotetext{
${ }^{4}$ See Section 6 for a discussion and comparison of techniques for proving lower bound on energy complexity.
} 
Theorem 1.3. Let $f:\{0,1\}^{n} \rightarrow\{0,1\}$ be a monotone function. Then $\mathrm{EC}(f)=\Omega\left(\mathrm{KW}^{+}(f)\right)$.

It is known that for the perfect matching function of a graph on $n$ edges, denoted as $f_{P M}$, $\mathrm{KW}^{+}\left(f_{P M}\right)=\Omega(\sqrt{n})$ [RW92]. Hence, Theorem 1.3 implies that any Boolean circuit with bounded fan-in, computing $f_{P M}$ will require energy at least $\Omega(\sqrt{n})$.

All the models considered so far are of fan-in 2. We now relax this requirement and consider the energy complexity of unbounded fan-in constant depth Boolean circuits computing specific functions. In this direction, we show the following.

Theorem 1.4. Let $C$ be any unbounded fan-in Boolean circuit of depth 3 computing the parity function on $n$ variables. Then, $\mathrm{EC}(C)$ is $\Omega(n)$.

Finally, we show lower bounds on the energy complexity of Boolean functions when restricted to Boolean formulas (instead of Boolean circuits), in terms of its formula size and depth.

For a formula $F$, let $\mathrm{L}(F)$ be the number of leaves in $F$ and Depth $(F)$ be the length of the longest path from root to any leaf in $F$. For a Boolean function $f$, let $L(f)$ be the minimum $L(F)$ among all the formulas $F$ computing $f$. Let $\operatorname{EC}^{\mathrm{F}}(f)$ be the minimum energy for any bounded fan-in formula computing $f$. Intuitively, Boolean formulas can take more energy than a Boolean circuit since we cannot "reuse" computation. Also, for any formula $F, \mathrm{EC}^{\mathrm{F}}(F) \leq \mathrm{L}(F)-1$. Hence, it would not be surprising if $\mathrm{EC}^{\mathrm{F}}(F)$ is also lower bounded by $\Omega(\mathrm{L}(F))$ giving a tight bound of $\mathrm{EC}^{\mathrm{F}}(f)=\Theta(\mathrm{L}(f))$. Towards this direction we show the following result.

Theorem 1.5. For a Boolean function $f$, computed by a formula $F$,

$$
\mathrm{EC}^{\mathrm{F}}(F)=\Omega(\sqrt{\mathrm{L}(F)}-\operatorname{Depth}(F))
$$

Related work: We discuss recent results on energy complexity of computing Boolean functions in various circuit models.

Observe that any Boolean circuit is also a threshold circuit since each of gates in $\mathcal{B}, \wedge, \vee$ and $\neg$ can be implemented by threshold gates. This implies that, $\mathrm{EC}(f) \geq \mathrm{EC}_{\mathcal{T}}(f)$. Hence, for a function $f$, known lower bound on $\mathrm{EC}_{\mathcal{T}}(f)$ translates to a lower bound on $\mathrm{EC}(f)$. In this context, Table 1 summarizes known results on bounds on energy complexity of threshold circuits in terms of the parameters size, depth and fan-in for certain classes of Boolean functions. For designing energy efficient circuits, techniques or tools to reduce the energy complexity of circuits is relevant in this context. Table 2 summarizes known results on energy complexity of Boolean functions on ways to transform circuits to energy efficient ones.

Energy vs circuit parameters: Table 1 presents the information: "Energy vs Parameter (Param) trade-off for any circuit $C$ using specific type of gates (Gate) computing the function $f^{\prime \prime}$. The parameters involved are $s=\operatorname{Size}(f), d=\operatorname{Depth}(f), \ell=$ fanin of gates in $C$ and $e$ is the optimum energy of a circuit with gates of type Gate computing $f$.

By $R_{\delta}(f)$, we denote the two-sided error public coin randomized communication complexity of $f$ with error probability $\delta$. We now describe the two notations $a_{f}$ and $b_{f}$ used in first two entries of Table 1 for a symmetric function $f$. Observe that any symmetric function $f:\{0,1\}^{n} \rightarrow\{0,1\}$ can be completely described by an $n+1$ length Boolean vector $-v_{f}$ as $f(x)=v_{f}(|x|)$ for all $x \in\{0,1\}^{n}$ where $|x|$ is the number of ones in $x$. For $b \in\{0,1\}$, let $g_{b}$ is maximum length 


\begin{tabular}{l|c|c|lc}
\hline Param & Function $f$ is ... & Gate & Trade-off & Ref. \\
\hline$\ell$ & Symmetric & any & $\ell \geq \frac{n-b_{f}}{e}$ & [SUZ13] \\
$s$ & Symmetric & Unate & $s^{e} \geq \frac{n+1-a_{f}}{b_{f}}$ & [UTN11] \\
$d, s$ & any & Threshold & $R_{0.5-\delta}(f)=O\left(e^{d} \log s\right)$, & [UT08] \\
& & & $\delta=\frac{1}{s^{O\left(e^{d}\right)}}$ & \\
\hline
\end{tabular}

Table 1: Known bounds on energy e of circuits computing Boolean functions

of consecutive $b^{\prime}$ s in $v_{f}$. Then, $a_{f} \stackrel{\text { def }}{=} \min \left\{g_{0}, g_{1}\right\}$ and $b_{f} \stackrel{\text { def }}{=} \max \left\{g_{0}, g_{1}\right\}$. The first two entries of Table 1 gives an energy fan-in trade-off and size energy trade-off, respectively, for symmetric functions in terms of $a_{f}$ and $b_{f}$. The third entry of Table 1 gives an energy size trade-off for constant depth threshold circuits for any Boolean function.

Energy of circuits under change of basis Table 2 presents the information: "Given a circuit $C$ with Energy $(C)=e$ of with gates of type $A$ then, there exists a circuit $C^{\prime}$ with gates of type $B$ computing the same function as $C$ with bounds on $\operatorname{Size}\left(C^{\prime}\right)$, Depth $\left(C^{\prime}\right)$, Energy $\left(C^{\prime}\right) . "$

\begin{tabular}{llllll}
\hline$A$ & $B$ & $\operatorname{Size}\left(C^{\prime}\right)$ & $\operatorname{Depth}\left(C^{\prime}\right)$ & $\operatorname{Energy}\left(C^{\prime}\right)$ & Ref. \\
\hline Any & Threshold & $\leq O((e+n) \operatorname{Size}(C))$ & $O(e)$ & - & [UNT10] \\
Threshold/Unate & Threshold/Unate & $\leq 2 \cdot e \cdot \operatorname{Size}(C)+1$ & $\leq 2 \cdot e+1$ & $e$ & [UNT10] \\
\hline
\end{tabular}

Table 2: Transforming circuit of type $A$ to type $B$

Organization of the paper. The rest of the paper is organized as follows. We start with preliminaries in Section 2. We show new bounds on energy complexity in terms of the decision tree depth in Section 3. Then, we show two methods to obtain lower bounds on energy complexity in Section 4.1 and Section 4.2 using the notion of continuous positive paths (introduced in Section 4.1.1). In Section 4.3, we show energy lower bounds for depth 3 Boolean circuits computing a specific function. Following this, in Section 5, we show energy lower bounds for Boolean formulas. In Section 6, we compare of our lower bound techniques with a recent improvement due to Sun et al. [SSWX19]. We conclude in Section 7 outlining some directions for further exploration.

\section{Preliminaries}

A Boolean circuit $C$ over the basis $\mathcal{B}=\left\{\wedge_{2}, \vee_{2}, \neg\right\}$ is a directed acyclic graph (DAG) with a root node (of out-degree zero), input gates labeled by variables (of in-degree zero) and the non-input gates (inclusive of root) labeled by functions in $\mathcal{B}$. Define the size to be the number of non-input gates and, depth to be the length of the longest path from root to any input gate of the circuit $C$ denoted, respectively, as $\operatorname{Size}(C)$ and $\operatorname{Depth}(C)$. A Boolean formula is a Boolean circuit where the underlying DAG is a tree. We call a negation gate that takes input from a variable as a leaf negation. A Boolean circuit is said to be monotone if it does not use any negation gates. A function is monotone if it can be computed by a monotone circuit. Equivalently, a function $f$ is monotone if 
$\forall x, y \in\{0,1\}^{n}, x \prec y \Longrightarrow f(x) \leq f(y)$ where $x \prec y$ iff $x_{i} \leq y_{i}$ for all $i \in[n]$. For a Boolean circuit $C$, negs $(C)$ denotes the number of NOT gates in the circuit $C$. Fix an arbitrary ordering among the gates of $C$. A firing pattern of a circuit $C$ on a given input is the binary string of evaluation of the gates on the input as per the fixed ordering. The number of firing patterns of a circuit $C$ is the number of distinct firing patterns for $C$ over all inputs.

For $i \in[n]$, let $e_{i}$ denote the $n$ length Boolean vector with the $i^{\text {th }}$ entry alone as 1 . For an $a \in\{0,1\}^{n}, a \oplus e_{i}$ denotes the input obtained by flipping the $i^{\text {th }}$ bit of $a$. The positive sensitivity of $f$ on $a$, denoted by psens $(f, a)$, is the number of $i \in[n]$ such that $a_{i}=1$ and $f\left(a \oplus e_{i}\right) \neq f(a)$. We define $\operatorname{psens}(f)$ as $\max _{a \in\{0,1\}^{n}} \operatorname{psens}(f, a)$.

For a monotone function $f:\{0,1\}^{n} \rightarrow\{0,1\}, x \in f^{-1}(1)$ and $y \in f^{-1}(0)$, define $S_{f}^{+}(x, y)=$ $\left\{i \mid x_{i}=1, y_{i}=0, i \in[n]\right\}$. The monotone Karchmer-Wigderson cost of $f$ (denoted by $\mathrm{KW}^{+}(f)$ ) is the optimal communication cost of the problem where Alice has $x$, Bob has $y$ and they have to find an $i \in[n]$ such that $i \in S_{f}^{+}(x, y)$. It is known that $\mathrm{KW}^{+}(f)$ equals the minimum depth monotone Boolean circuit computing $f$. For more details about this model, see [KN06]. We now define the tree function - Definition 3.1 of [DS18].

Definition 2.1 (Tree Function). Let $\mathcal{F}=\left\{f_{k} \mid k \in \mathbb{N}\right\}$ be a family of Boolean functions where for every $k \in \mathbb{N}, f_{k}:\{0,1\}^{2^{k}-1} \rightarrow\{0,1\}$ is defined by the decision tree which is a full binary tree of depth $k$ with each of the $2^{k}-1$ internal node querying a distinct variable and each of the nodes at level $k$ have left leaf child labeled 0 and right leaf child labeled 1.

Energy Complexity: For a Boolean circuit $C$ and an input $a$, the energy complexity of $C$ on the input $a$ (denoted by $\operatorname{EC}(C, a)$ ) is defined as the number of non-input gates that output a 1 in $C$ on the input $a$. Define the energy complexity of $C$ (denoted by $\operatorname{EC}(C)$ ) as $\max _{a} \operatorname{EC}(C, a)$. The energy complexity of a function $f$, (denoted by $\mathrm{EC}(f)$ ) is the energy of the minimum energy circuit over the Boolean basis $\mathcal{B}$ computing $f$.

As mentioned in the introduction, Lozhkin and Shupletsov [LS15] showed that $\mathrm{EC}(f) \leq 3 n(1+$ $\epsilon(n))$ by constructing a Boolean circuit of size $\frac{2^{n}}{n}(1+\epsilon(n))$ where $\epsilon(n) \rightarrow 0$ as $n \rightarrow \infty$. Their idea is to construct a Boolean circuit of low energy that outputs all product terms on $n$ variables where each of them appears exactly once in a negated or unnegated form. We call such terms as minterms. We slightly improve the above bound using the same idea by constructing a Boolean circuit of size $2^{O(n)}$.

Proposition 2.2. For any $f:\{0,1\}^{n} \rightarrow\{0,1\}, \mathrm{EC}(f) \leq 3 n-1$.

Proof. We show that all minterms in $n$ variables can be computed by a Boolean circuit of energy at most $2 n-1$. Assuming this, to compute $f$, construct an $\vee$ formula on $2^{n}$ inputs of depth $n$ and connect the minterms on which $f$ evaluates to 1 as the leaves of $\vee$ (and the rest of the inputs as 0 ). Since on any input, exactly one of the leaves will evaluate to 1 , there is only 1 path to the output gate where all $\wedge$ gates evaluate to 1 . Hence, the overall energy complexity is at most $2 n-1+n=3 n-1$. We construct a Boolean circuit of energy $2 n-1$ to compute all minterms on $n$ variables.

Proof is by induction on $n$. Let $x_{1}, \ldots, x_{n}$ be the variables. For $n=1$, the Boolean circuit is $x_{1}$, $\neg x_{1}$ which has energy 1 . Hence, the base case holds.

By induction, we have constructed a circuit $C$ (on $n$ inputs and having $2^{n}$ outputs) computing all $2^{n}$ minterms on $x_{1}, \ldots, x_{n}$. We modify the Boolean circuit as follows : branch out each output 
gate into two (left and right branch). Connect the left (resp. right) branch output to $x_{n+1}$ (resp. $\neg x_{n+1}$ ) by an $\wedge$ gate. Note that out of all $2^{n+1}$ outputs created this way, exactly one of them will output 1 on any input. Also we have computed all $2^{n+1}$ minterms on $x_{1}, \ldots, x_{n+1}$. The resulting circuit has an energy of $2 n-1$ for circuit $C$ by induction plus 2 due to the output and the negation gate of $x_{n+1}$. Hence, the overall energy is $2 n+1=2(n+1)-1$. This completes the induction.

The upper bound in Proposition 2.2 has been improved by Sun et al. [SSWX19] from $3 n-1$ to $3 n-2$.

Observe that in a Boolean circuit $C$, for the leaf negation gates, there is always an input where all of them output a 1 . For the non-leaf negation gates, irrespective of the input, either the negation gate or its input gate will output a one. Due to this reason, we have,

Proposition 2.3. For any circuit $C, \mathrm{EC}(C) \geq \operatorname{negs}(C)$.

Model specific variants of energy complexity: We now consider the notion of energy complexity for three other circuit models, namely monotone circuits, Boolean formulas and threshold circuits.

Energy Complexity and Monotone Boolean circuits: For a monotone Boolean function $f$, computed by a monotone Boolean circuit $C$, define $\mathrm{EC}^{\mathrm{M}}(C)$ as the maximum over all the inputs the number of non-input gates that output a 1 . We define $\mathrm{EC}^{\mathrm{M}}(f)$ as $\min _{C} \mathrm{EC}^{\mathrm{M}}(C)$ where $C$ is a monotone circuit computing $f$. The following proposition gives an exact characterization for $\operatorname{EC}^{\mathrm{M}}(f)$.

Proposition 2.4. For a monotone Boolean function $f$, let $\mathrm{mSize}(f)$ denotes the size of the smallest monotone Boolean circuit computing $f$. Then, $\mathrm{EC}^{\mathrm{M}}(f)=\mathrm{mSize}(f)$.

Proof. Let $C$ be a monotone Boolean circuit of minimum size computing $f$. Clearly, $\mathrm{EC}^{\mathrm{M}}(f) \leq$ $\mathrm{EC}^{\mathrm{M}}(C) \leq \mathrm{mSize}(f)$. Also, for any monotone circuit $C^{\prime}$ computing $f$, on the input $1^{n}$, all the gates in $C^{\prime}$ output a 1 implying $\mathrm{EC}^{\mathrm{M}}\left(C^{\prime}\right) \geq \operatorname{EC}^{\mathrm{M}}\left(C^{\prime}, 1^{n}\right)=\operatorname{mSize}\left(C^{\prime}\right)$. In particular, for the monotone circuit $C^{\prime \prime}$ of minimum energy computing $f, \operatorname{EC}^{\mathrm{M}}(f)=\operatorname{EC}^{\mathrm{M}}\left(C^{\prime \prime}\right) \geq \operatorname{Size}\left(C^{\prime \prime}\right) \geq \operatorname{mSize}(f)$. Hence, $\mathrm{EC}^{\mathrm{M}}(f)=\operatorname{mSize}(f)$.

Energy Complexity and Threshold circuits: Let $\mathcal{T}$ be a basis consisting of all weighted threshold functions. A threshold circuit is a Boolean circuit where the gates are from the basis $\mathcal{T}$. Uchizawa et al. [UDM06] introduced the notion of energy complexity of threshold circuits denoted by $\mathrm{EC}_{\mathcal{T}}(C)$, again defined as the worst energy of the threshold circuit $C$ among all the inputs. Define $\mathrm{EC}_{\mathcal{T}}(f)$ as $\min _{C} \mathrm{EC}_{\mathcal{T}}(C)$ where $C$ is a threshold circuit over the basis $\mathcal{T}$ computing $f$.

A decision tree is a rooted tree with all the non-leaf nodes labeled by variables and leaves labeled by a 0 or 1 . Note that every assignment to the variable in the tree defines a unique path from root to leaf in the natural way. A Boolean function $f$ is said to be computed by a decision tree if for every input $a$, the path from root to a leaf guided by the input is labeled by $f(a)$. Depth of a decision tree is the length of the longest path from root to any leaf. Define decision tree depth of $f$ (denoted by DT $(f)$ ) as the depth of the minimum depth decision tree computing $f$. A threshold decision tree is similar to the decision tree except that queries at each non-leaf node can be an arbitrary threshold function on the input variables. We denote the depth of the minimum depth threshold decision tree computing $f$ by $\mathrm{DT}_{\text {th }}(f)$.

For an $f:\{0,1\}^{n} \rightarrow\{0,1\}$, Uchizawa et al. [UDM06] introduced a measure of energy for threshold decision tree $T$ computing $f$, denoted by $\cos t(T)$ defined as the maximum over all paths 
from root to leaf, the number of right turns taken in a path with cost of leaf defined to be zero. As mentioned in [UDM06], cost $(T)$ can be seen as a measure of how often does a threshold gate in $T$ output a 1 .

As a part of their main result they showed, given any threshold decision tree $T$, (1) how to construct another threshold decision tree $T^{\prime}$ with a bound on $\operatorname{cost}\left(T^{\prime}\right)$ (Lemma 2, 3 of [UDM06]) and (2) how to obtain a low energy threshold circuit $C^{\prime}$ computing $f$ from $T$ of energy $\operatorname{cost}(T)$. (Lemma 5 of [UDM06]).

This implies the following relation between $\mathrm{EC}_{\mathcal{T}}(f)$ and $\mathrm{DT}_{t h}(f)$.

Proposition 2.5. For any Boolean function $f, \mathrm{EC}_{\mathcal{T}}(f) \leq \mathrm{DT}_{\text {th }}(f)+1$.

Proof. Let $T$ be an optimum threshold decision tree computing $f$ with depth $\mathrm{DT}_{t h}(f)$. We first state the results (1) and (2) formally. Result (1) says that $f$ can be computed by another threshold decision tree $T^{\prime}$ with same depth as $T$, same number of leaves as $T$ and have $\operatorname{cost}\left(T^{\prime}\right) \leq$ $\log (\#$ leaves of $T$ ) (Lemma 2, 3 of [UDM06]). Result (2) says that there exists a threshold circuit $C$ computing $f$ with $\mathrm{EC}_{\mathcal{T}}(C) \leq \operatorname{cost}(T)+1$ (Lemma 5 of [UDM06]).

Since $T$ has at most $2^{\mathrm{DT}_{t h}(f)}$ many leaves, applying (1), we get a threshold decision tree $T^{\prime}$ with $\operatorname{cost}\left(T^{\prime}\right) \leq \log (\#$ leaves of $T) \leq \mathrm{DT}_{t h}(f)$. The result now follows by applying (2) to $T^{\prime}$.

Energy Complexity and Formulas: For a Boolean formula $F$, define $\operatorname{EC}^{\mathrm{F}}(F)$ is the worst case energy complexity of the formula $F$ over the Boolean basis $\mathcal{B}$. We define, $\operatorname{EC}^{\mathrm{F}}(f)$ as $\min _{F} \operatorname{EC}^{\mathrm{F}}(F)$ where $F$ is formula (over the Boolean basis $\mathcal{B}$ ) computing $f$. See Section 5 for more details.

In the rest of the sections, by circuits, we refer to Boolean circuits over the Boolean basis $\mathcal{B}$.

\section{Energy Complexity and Decision Trees}

In this section, we show a new technique to obtain upper bounds on $\mathrm{EC}(f)$.

Recall that any $n$ bit function $f$ can be computed by a circuit of energy at most $3 n-1$ (Proposition 2.2). In this section, we identify the property of having low depth decision trees as a sufficient condition to guarantee energy efficient circuits. More precisely, we show that for any Boolean function $f, \mathrm{EC}(f) \leq \mathrm{O}\left(\mathrm{DT}(f)^{3}\right)$.

One of the challenges in constructing a Boolean circuit is to use as few negation gates as possible. The reason is that non-leaf negation gates always contribute to the energy since either the gate or its input will always output a 1 on any input to the circuit. We achieve this in our construction via an idea inspired by the connector circuit introduced by Markov [Mar58]. Before describing the construction, we need the following result (Lemma 3.1) which helps in controlling the number of negation gates in our construction.

Lemma 3.1. Let $f_{0}$ and $f_{1}$ be any two Boolean functions on $n$ variables computed by Boolean circuits $C_{0}$ and $C_{1}$ respectively. Fix an $i \in[n]$. Define $f(x)=\left(\neg x_{i} \wedge f_{0}(x)\right) \vee\left(x_{i} \wedge f_{1}(x)\right)$. Then, a circuit $C$ computing $f$ can be obtained using $C_{0}$ and $C_{1}$ such that $\operatorname{negs}(C)=1+\max \left\{\operatorname{negs}\left(C_{0}\right), \operatorname{negs}\left(C_{1}\right)\right\}$.

Note that the existence of the circuit in Lemma 3.1 can also be argued using the result of Markov [Mar58] (see Section 10.2 of Jukna [Juk12]) for an arbitrary $f$. However, the construction obtained by directly using the result of Markov can potentially have high energy and hence is not suitable in our context. Since the Boolean function $f$ which we intent to compute is structured, we take advantage of this observation to adapt Markov's construction and obtain a low energy 
circuit (with minimal number of negation gates) in Lemma 3.1 which is then used to prove the main result of this section (Theorem 1.1).

Proof of Lemma 3.1. We start with the circuit $A=\left(\neg x_{i} \wedge C_{0}(x)\right) \vee\left(x_{i} \wedge C_{1}(x)\right)$ which uses $1+$ negs $\left(C_{0}\right)+\operatorname{negs}\left(C_{1}\right)$ negations to compute $f$. If one of negs $\left(C_{0}\right)$ or negs $\left(C_{1}\right)$ is zero, then $A$ is the required circuit. Otherwise, we modify this circuit in $\min \left\{\operatorname{negs}\left(C_{0}\right)\right.$, negs $\left.\left(C_{1}\right)\right\}$ steps where, in each step, we reduce the number of negations by 1 such that the resulting circuit computes $f$ correctly. Hence the resulting circuit $C$ has $1+\operatorname{negs}\left(C_{0}\right)+\operatorname{negs}\left(C_{1}\right)-\min \left\{\operatorname{negs}\left(C_{0}\right), \operatorname{negs}\left(C_{1}\right)\right\}=$ $1+\max \left\{\operatorname{negs}\left(C_{0}\right), \operatorname{negs}\left(C_{1}\right)\right\}$ negations.

We describe the modifications starting with $A$. Let $g_{0}$ be a gate in $C_{0}$ that feeds into a negation gate such that the function computed at $g_{0}$ does not depend on the output of any negation gate. Let $D_{0}$ be the sub-circuit rooted at $g_{0}$. Similarly, let $g_{1}$ be a gate in $C_{1}$ with the similar property and let $D_{1}$ be the sub-circuit rooted at $g_{1}$. We remove the negation gates that $g_{0}$ and $g_{1}$ feeds into from $C_{0}$ and $C_{1}$ respectively and construct the connector circuit (as shown in the box in Fig. 1a). We feed the output from the selector as output of the negation gates in $C_{0}$ and $C_{1}$. Let $D_{0}^{\prime}$ (resp. $D_{1}^{\prime}$ ) be the circuit $C_{0}$ (resp. $C_{1}$ ) with the output of selector circuit acting as output of the negation gate with the negation gate alone removed (Note that we do not completely disconnect the sub-circuits from the circuit. The wires connecting $D_{0}$ (resp. $D_{1}$ ) to $D_{0}^{\prime}$ (resp. $D_{1}^{\prime}$ ) are not shown in Fig. 1a to avoid clutter).

When $x_{i}=0$, we claim that this circuit outputs $C_{0}(x)$. This is because when $x_{i}=0, D_{0}^{\prime}$ gets $\neg D_{0}$ as output of $g_{0}$ correctly and hence computes $C_{0}(x)$ while the output of $D_{1}^{\prime}$ is inhibited by the $\wedge$ gate which it feeds into. By a similar argument, this circuit computes $C_{1}(x)$ when $x_{i}=1$. Hence the resulting circuit indeed computes $f$ correctly.

Observe that the number of negation goes down by one in each step since we replace two negations by one. We repeat the previous steps restricted to gates in $D_{0}^{\prime}$ and $D_{1}^{\prime}$ as long as the negations in at least one of the circuits is exhausted. By the earlier argument, the final circuit $C$ correctly computes $f$.

When $x_{1}=0$ (resp. $x_{1}=1$ ) the part of the circuit computing $f_{1}$ (resp. $f_{0}$ ) is not necessary in computing $f$. Having obtained a circuit construction which minimizes the usage of negations, we need a way to "turn off" such gates that are not needed in computing $f$. In Lemma 3.2, we demonstrate how this is achieved, thereby saving energy, at the cost of increasing the fan-in of $\wedge$ gates.

Lemma 3.2. For any non-constant Boolean function $f$, there exists a circuit $C$ computing $f$ with, (1) all $\vee$ gates are of fan-in 2 and all $\wedge$ gates are of fan-in at most $\mathrm{DT}(f)+2,(2)$ no $\vee$ gate have a negation gate or a variable directly as its input, (3) negs $(C) \leq \mathrm{DT}(f)$ and, (4) $\mathrm{EC}(C) \leq 2 \mathrm{DT}(f)^{2}$.

Proof. We describe the construction of the circuit by an induction on DT $(f)$.

Base Case: For $f$ with $\mathrm{DT}(f)=1, f$ is either a variable or its negation and hence the trivial circuit satisfies (1) to (4). For DT $(f)=2$, let $T$ be an optimal decision tree with $x_{1}$ as its root. Then, $f$ can be computed by the circuit $C=\left(\neg x_{1} \wedge \ell_{1}\right) \vee\left(x_{1} \wedge \ell_{2}\right)$ where $\ell_{1}, \ell_{2}$ could be a variable, negation of a variable or a constant. Also, if $C$ has 3 negations, we apply Lemma 3.1 to get a circuit with two negations. Hence, condition (3) is satisfied. In either cases, the conditions (1) and (2) are also satisfied and it can be verified that the energy of the circuit is at most $5 \leq 2 \mathrm{DT}(f)^{2}$. Hence, condition (4) is also satisfied and the base case holds. 


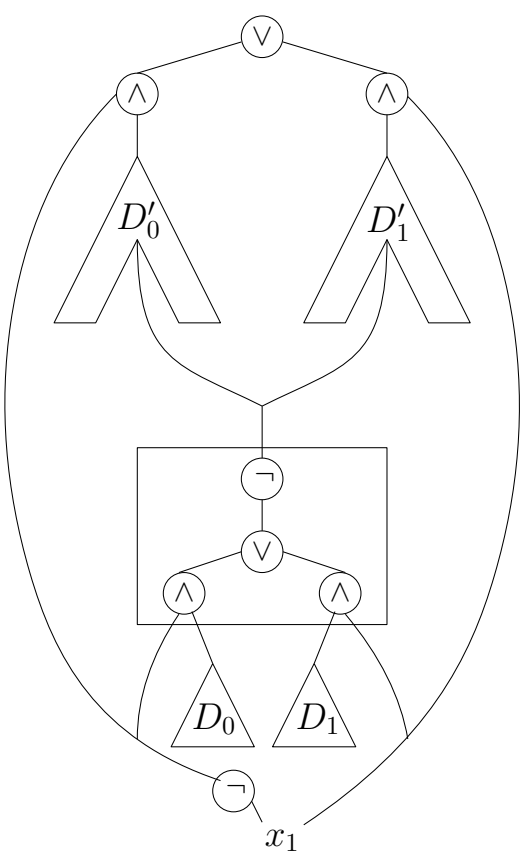

(a) First step in the construction of $C$ in the proof of Lemma 3.1.

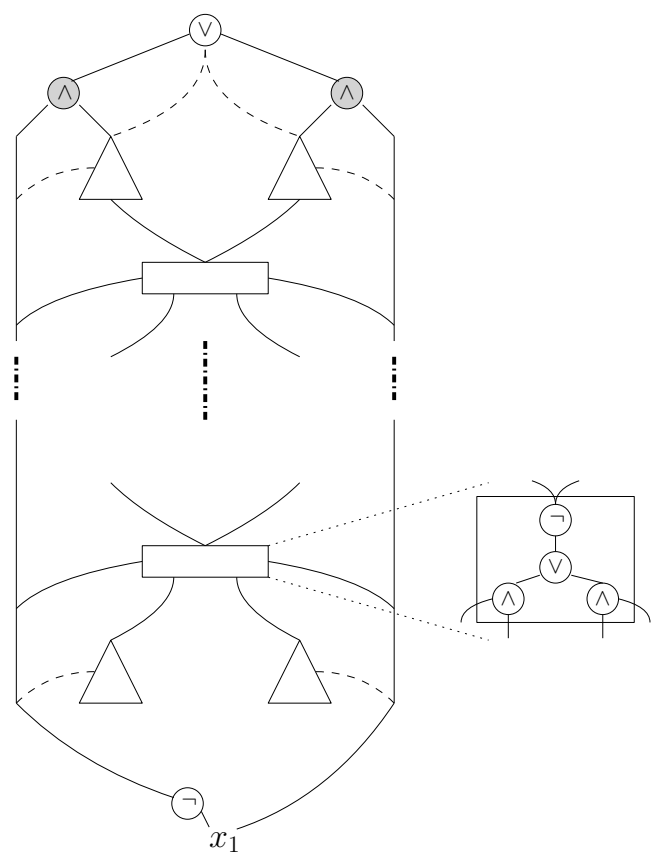

(b) Circuit $C^{\prime}$ in the proof of Lemma 3.2

Figure 1: Energy efficient circuit construction

Inductive Step: Let $f$ be a Boolean function computed by a decision tree $T$ of depth DT $(f) \geq 3$. By induction, assume that for any Boolean function $g$ with DT $(g) \leq \mathrm{DT}(f)-1$ there exists a circuit $C$ computing $g$ satisfying (1) to (4). Let the root variable of $T$ be $x_{1}$ and $T_{0}$ (resp. $T_{1}$ ) be the left (resp. right) subtree computing the function $f_{0}=\left.f\right|_{x_{1}=0}$ (resp. $\left.f_{1}=\left.f\right|_{x_{1}=1}\right)$. Since $f_{0}$ and $f_{1}$ are computed by decision trees of depth $\mathrm{DT}(f)-1$, by induction, there exists circuits $C_{0}$ and $C_{1}$ computing $f_{0}$ and $f_{1}$, respectively, satisfying (1) to (4).

Observe that $f(x)=\left(\neg x_{1} \wedge f_{0}\right) \vee\left(x_{1} \wedge f_{1}\right)$. Hence by Lemma 3.1, there exists a circuit $C$ computing $f$ (Fig. $1 \mathrm{~b}$ omitting the thinly dashed lines) with negs $(C)=\max \left\{\operatorname{negs}\left(C_{0}\right), \operatorname{negs}\left(C_{1}\right)\right\}+$ 1. We modify the circuit $C$ as follows: for each $\wedge$ gate which was originally in $C_{0}$ (resp. $C_{1}$ ), we add $\neg x_{1}$ (resp. $x_{1}$ ) as input thereby increasing its fan-in by 1 . We also remove the $\wedge$ gate (shaded in Fig. 1b) feeding into the top $\vee$ gate and feed the output of the circuits directly to the top $\vee$ gate (shown as dashed in Fig. 1b). Call the resulting circuit $C^{\prime}$ and the gates from $C_{0}$ as $C_{0}^{\prime}$ (the left part in Fig. 1b) and the gates from $C_{1}$ as $C_{1}^{\prime}$ (the right part in Fig. 1b).

We first argue that the conditions (1) and (2) holds true for $C^{\prime}$. We then argue that $C^{\prime}$ correctly computes $f$ using which we argue (3) and (4) thereby completing the induction.

We observe that the condition (1) holds since $\vee$ gate has fan-in 2 by construction and $\wedge$ gate has fan-in at most max $\left\{\mathrm{DT}\left(f_{0}\right)+3, \mathrm{DT}\left(f_{1}\right)+3\right\}$ which is at most $\mathrm{DT}(f)+2$. The removal of the shaded $\wedge$ gates never causes a variable or a negation to be fed to the top $\vee$ gate since $f_{0}$ and $f_{1}$ have a decision tree depth of at least 2 and hence the circuits of the respective functions have top gate as $\vee$ which is guaranteed by base case for depth 2 and by induction otherwise. Hence, the condition (2) holds. We now argue that $C^{\prime}$ correctly computes $f$. When $x_{1}=1$, all the $\wedge$ gates in $C_{0}^{\prime}$ evaluate to 0 . Since no input variable or negation gate feeds into any $\vee$ gate in $C_{0}^{\prime}$ (condition (2)), 
all the $\vee$ gates and $\wedge$ gates output 0 irrespective of the remaining input bits. Hence, $C_{0}^{\prime}$ outputs 0 . Since $x_{1}=1, C_{1}^{\prime}$ behaves exactly same as $C_{1}$. By Lemma 3.1, the circuit $C_{1}$ correctly computes $f$ when $x_{1}=1$. Hence, the circuit $C^{\prime}$ correctly computes $f$ for $x_{1}=1$. The same argument with $C_{0}$ and $C_{1}$ interchanged can be used to show that $C^{\prime}$ correctly computes $f$ with $x_{1}=0$.

Since no new negations were added in $C^{\prime}$, negs $\left(C^{\prime}\right)=\operatorname{negs}(C)$ which, by Lemma 3.1, equals $\max \left\{\operatorname{negs}\left(C_{0}\right), \operatorname{negs}\left(C_{1}\right)\right\}+1 \leq \max \left\{\mathrm{DT}\left(f_{1}\right), \mathrm{DT}\left(f_{2}\right)\right\}+1 \leq \mathrm{DT}(f)$. Hence the condition (3) holds. We show that condition (4) holds for $C^{\prime}$. Let $x$ be an input with $x_{1}=1$. We have already argued that when $x_{1}=1$, none of the $\wedge$ or $\vee$ gates of $C_{0}^{\prime}$ output a 1 . Hence the gates that can output a 1 in $C^{\prime}$ are the negations in $C_{0}^{\prime}$, the gates that output 1 in $C_{1}^{\prime}$, the selector gates (in the construction of Lemma 3.1), the root gate and the negation gate for $x_{1}$ (recall that the shaded $\wedge$ gates are removed). Observe that the negations in $C_{0}^{\prime}$ is at most negs $\left(C_{0}\right)$ and $C_{1}^{\prime}$ behaves exactly as $C_{1}$ for $x_{1}=$ 1. Also, the number of selector circuits used in Lemma 3.1 is at most $\max \left\{\operatorname{negs}\left(C_{0}\right), \operatorname{negs}\left(C_{1}\right)\right\} .{ }^{5} \mathrm{In}$ addition, each such circuit can have at most 2 gates that output 1 on any input (see Fig. 1b). Hence, $\mathrm{EC}\left(C^{\prime}, x\right) \leq \alpha_{0}=\operatorname{negs}\left(C_{0}\right)+\mathrm{EC}\left(C_{1}\right)+2 \max \left\{\operatorname{negs}\left(C_{0}\right), \operatorname{negs}\left(C_{1}\right)\right\}+2$. For $x$ with $x_{1}=0$, by a similar argument, $\mathrm{EC}\left(C^{\prime}, x\right) \leq \alpha_{1}=\operatorname{negs}\left(C_{1}\right)+\mathrm{EC}\left(C_{0}\right)+2 \max \left\{\operatorname{negs}\left(C_{0}\right), \operatorname{negs}\left(C_{1}\right)\right\}+2$. Hence, $\mathrm{EC}\left(C^{\prime}\right) \leq \max \left\{\alpha_{0}, \alpha_{1}\right\}$ which is at most $\max \left\{\mathrm{EC}\left(C_{0}\right), \mathrm{EC}\left(C_{1}\right)\right\}+3 \max \left\{\operatorname{negs}\left(C_{0}\right), \operatorname{negs}\left(C_{1}\right)\right\}+2$. By induction, we have $\mathrm{EC}\left(C^{\prime}\right) \leq 2(\mathrm{DT}(f)-1)^{2}+3(\mathrm{DT}(f)-1)+2$ which implies $\mathrm{EC}\left(C^{\prime}\right) \leq$ $2 \mathrm{DT}(f)^{2}$ as $f$ is non-constant. This completes the induction.

We prove the main result of this section.

Theorem 1.1. For any Boolean function $f, \mathrm{EC}(f) \leq O\left(\mathrm{DT}(f)^{3}\right)$.

Proof. If $f$ is constant, the result holds. Otherwise, applying Lemma 3.2, we have a circuit $C^{\prime}$ computing $f$ with fan-in of $\vee$ gate being 2 and fan-in of $\wedge$ gate being at most DT $(f)+2$ of energy at most 2DT $(f)^{2}$. Without loss of generality, let $x_{1}$ be the variable at the root of the decision tree. By construction, all the unbounded fan-in $\wedge$ gates of the circuit $C^{\prime}$ have $x_{1}$ or $\neg x_{1}$ as an input.

To obtain a bounded fan-in circuit from $C^{\prime}$, we replace each of the $\wedge$ gates by a fan-in 2 circuit as follows. Let $g$ be a $\wedge$ gate of the circuit $C^{\prime}$ of fan-in $c \leq \mathrm{DT}(f)+2$ which takes in $\ell \in\left\{x_{1}, \neg x_{1}\right\}$ as one of its input. We replace $g$ by a tree of fan-in $2 \wedge$ gates of $c$ leaves and of depth $c-1$ with $\ell$ as a leaf at depth $c-1$ as shown in Fig. 2 .

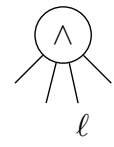

(a) $\wedge$ gate of fan-in $c$

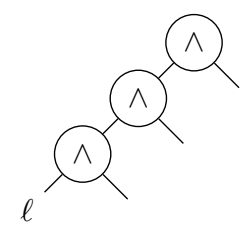

(b) Tree of depth $c-1$

Figure 2: Handling $\wedge$ gates of large fan-in $c($ for $c=4)$

We now argue that this replacement with $\ell$ reattached as the leftmost leaf can only increase the overall energy by a factor of at most $c-1$. Consider an input for which $\ell=0$. Then, irrespective of the values of other $c-1$ inputs, none of the fan-in $2 \wedge$ gates output a 1 as $\ell$ forces all $\wedge$ gates to output 0 . On the other hand if $\ell=1$, then the added gates can contribute an energy of at most

\footnotetext{
${ }^{5}$ While this quantity should be the minimum of the negations of $C_{1}$ and $C_{2}$, as seen in the proof of Lemma 3.1, we upper bound this by the maximum of negations.
} 
$c-1$. Hence, for any input, the $\wedge$ gates in $C^{\prime}$ that output a 0 does not contribute any energy and those that output a 1 can contribute of an energy of at most $c-1 \leq \mathrm{DT}(F)+1$. Since, in the worst case, all the gates that output a 1 can be an $\wedge$,

$$
\mathrm{EC}(f) \leq \mathrm{EC}(C) \cdot(\mathrm{DT}(f)+1) \leq 2 \mathrm{DT}(f)^{2} \cdot(\mathrm{DT}(f)+1)=\mathrm{O}\left(\mathrm{DT}(f)^{3}\right)
$$

\section{Lower Bounds on Energy Complexity}

In this section, we introduce new methods to show lower bounds on energy complexity of Boolean functions. We introduce the notion of continuous positive paths (Section 4.1.1) using which we prove two energy lower bounds. Firstly, we show that the positive sensitivity of a function is a lower bound on its energy complexity (Section 4.1.2). Secondly, we show that for monotone Boolean functions, the cost of the monotone Karchmer- Wigderson game for the function is a lower bound on its energy complexity (Section 4.2). We conclude the section by proving an energy lower bound of $\Omega(n)$ for any depth 3 unbounded fan-in circuit computing parity function on $n$ bits (Section 4.3).

\subsection{Energy Lower Bounds from Positive Sensitivity}

In this section, we prove Theorem 1.2 from the introduction. We first describe an outline here. As a starting case, consider a monotone circuit $C$ computing $f$ evaluates to 1 on an input $a \in\{0,1\}^{n}$. Let $i \in[n]$ be such that $a_{i}=1$ and flipping $a_{i}$ to 0 causes the circuit to evaluate to 0 . We show that for such an index $i$ on input $a$, there is a path from $x_{i}$ to the root such that all the gates in the path outputs a 1. The latter already implies a weak energy lower bound. We then generalize this idea to non-monotone circuits as well and use it to prove energy lower bounds. This generalization also helps us to prove upper bounds for $\mathrm{KW}^{+}$games in Section 4.2.

To keep track of all input indices that are sensitive in the above sense, we introduce the measure of positive sensitivity denoted by psens $(f)$ (as defined in Section 2). For example, the functions $f \in\left\{\oplus_{n}, \wedge_{n}\right\}$ have psens $(f)=n$ while psens $\left(\vee_{n}\right)=1$. Let $\widetilde{\operatorname{psens}}(f, a)$ denote the set of positive sensitive indices on $a$.

\subsubsection{Continuous Positive Paths}

Let $C$ be a Boolean circuit computing $f:\{0,1\}^{n} \rightarrow\{0,1\}$. For an input $a \in\{0,1\}^{n}$, we call a path of gates such that every gate in the path output 1 on $a$ as a continuous positive path in $C$.

Fix an $a \in\{0,1\}^{n}$. We argue that for every positive sensitive index $i$ on $a$, either there is a continuous positive path from $x_{i}$ to the root or it must be broken by a negation gate of the circuit. Using this we show, in the next section, that energy complexity of a function is lower bounded by its positive sensitivity.

Lemma 4.1. Let $f:\{0,1\}^{n} \rightarrow\{0,1\}$ and $a \in\{0,1\}^{n}$ be an input such that $\operatorname{psens}(f, a) \neq 0$ and $i \in \widetilde{\operatorname{psens}}(f, a)$. Let $C$ be any circuit computing $f$. Then, either (1) there is a continuous positive path from $x_{i}$ to root or (2) $x_{i}$ directly feeds into a negation gate or (3) there is a continuous positive path from $x_{i}$ to $a$ gate which feeds into a negation gate of $C$. 
Proof. It suffices to prove the following stronger statement: for a Boolean function $f$ and an $a \in$ $\{0,1\}^{n}$ with $\operatorname{psens}(f, a) \neq 0$ and $i \in \overline{\operatorname{psens}}(f, a)$, let $C$ be any circuit such that $C(a)=f(a)$ and $C\left(a \oplus e_{i}\right)=f\left(a \oplus e_{i}\right)$. Then, either (1) there is a continuous positive path from $x_{i}$ to root or (2) $x_{i}$ directly feeds into a negation gate or (3) there is a continuous positive path from $x_{i}$ to a gate which feeds into a negation gate of $C$.

Proof is by induction on negs $(C)$. Let $C$ be any circuit such that $f(a)=C(a)$ and $f\left(a \oplus e_{i}\right)=$ $C\left(a \oplus e_{i}\right)$.

Base Case: For the base case, negs $(C)=0$ and $C$ is a monotone circuit. By definition, $i \in \operatorname{psens}(f, a)$ implies that $a>a \oplus e_{i}$. We claim that if $i \in \operatorname{psens}(f, a)$, then $C(a)=1$. For a contradiction, suppose that $f(a)=C(a)=0$. Then $C\left(a \oplus e_{i}\right)=0$ because $C$ is monotone. But then $f\left(a \oplus e_{i}\right)=0$ which contradicts the fact that $i \in \operatorname{psens}(f, a)$.

Since $C(a)$ outputs 1 and since $C$ is a monotone circuit, the root being an $\vee$ or $\wedge$ gate must have a child gate evaluating to 1 . Since this gate is again $\vee$ or $\wedge$ the same argument applies implying that there exists a series of gates all evaluating to 1 reaching some inputs. For any $i \in \operatorname{psens}(f, a)$, we show that there must a path from $x_{i}$ to the root with all the gates in the path evaluating to 1 in $C$ on input $a$ (implying that (1) holds).

For a contradiction, suppose that every path from $x_{i}$ to the root gate passes via some gate that evaluates to 0 . Among all the paths from $x_{i}$ to the root, collect all the gates that evaluate to 0 for the first time in the path and call this set as $T$. We fix all the variables except $x_{i}$ to the values in $a$ and view each of the gates in $g \in T$ as a function of $x_{i}$. Now, flipping $x_{i}$ from $a_{i}=1$ to 0 does not change the output of any $g \in T$ as they compute monotone functions and already evaluate to 0 . Since all other values are fixed, the output of the root gate does not change by this flip which contradicts the fact that $i \in \operatorname{psens}(f, a)$.

Induction Step: Let $C$ be a circuit with $f(a)=C(a)$ and $f\left(a \oplus e_{i}\right)=C\left(a \oplus e_{i}\right)$ and negs $(C) \geq 1$. If $x_{i}$ feeds directly into a negation gate, then statement (2) holds as required. Otherwise, let $g$ be the first gate that feeds into a negation in the topologically sorted order of the gates of $C$.

We have the following two possibilities. In both the cases, we argue existence of continuous positive path in $C$ from the variable $x_{i}$, thereby completing the induction.

- On input $a$, flipping $a_{i}$ change the output of $g$. Denote the function computed at $g$ as $f_{g}$. Then $f_{g}$ is monotone and $i \in \widehat{\operatorname{psens}}\left(f_{g}, a\right)$ and is non-empty. Hence, applying the argument in the base case to $f_{g}$ and the monotone circuit rooted at $g$, we are guaranteed to get a continuous positive path from $x_{i}$ to $g$. Since the circuit at $g$ is a sub-circuit of $C$ (that is, it appears as an induced subgraph), this gives a continuous positive path in $C$ also.

- On input $a$, flipping $a_{i}$ does not change the output of $g$. In this case, we remove the negation gate that $g$ feeds into and hard-wire the output of this negation gate (on input $a$ ) in $C$ to get a circuit $C^{\prime}$. Note that all other gates in $C$ are left intact. Observe that $C^{\prime}(a)=f(a)$. Since flipping $a_{i}$ did not change the output of $g$ and as all other gates are left intact, $C^{\prime}\left(a \oplus e_{i}\right)=$ $f\left(a \oplus e_{i}\right)$. As negs $\left(C^{\prime}\right)=\operatorname{negs}(C)-1$, by induction, either (1) there is a continuous positive path from $x_{i}$ to root or (2) there is a continuous positive path from $x_{i}$ to a gate which feeds into a negation gate of $C^{\prime}$. By construction, $C^{\prime}$ is same as $C$ except for the negation gate. Hence, a continuous positive path in $C^{\prime}$ is also a continuous positive path in $C$. 


\subsubsection{From Positive Sensitivity to Energy Lower Bounds}

We call the negation gates and the root gate of a circuit as target gates. In Lemma 4.1, we have already shown the existence of continuous positive paths from a positive sensitive index up to a target gate. Using this, we show an energy lower bound for any circuit of bounded fan-in computing a Boolean function $f$ in terms of $\operatorname{psens}(f)$. Since the fan-in of the circuit is limited, we exploit the idea that in a connected DAG, the number of internal nodes (in-degree at least 1) is lower bounded by the number of source nodes (in-degree 0 ).

Since every such positive sensitive index is reachable via a continuous positive path from a target gate, we obtain a lower bound on energy by applying this idea on an appropriate subgraph constructed from our circuit.

Theorem 1.2. For any Boolean function $f:\{0,1\}^{n} \rightarrow\{0,1\}$ computed by a circuit $C$ over the Boolean basis, $\mathrm{EC}(C) \geq \operatorname{psens}(f) / 3$.

Proof. Without loss of generality assume that $f$ is non-constant. Let $C$ be any circuit computing $f$ of fan-in 2 such that $\mathrm{EC}(C)=\mathrm{EC}(f)$. We prove that $\forall a \in\{0,1\}^{n}$, $\operatorname{psens}(f, a) \leq 3 \mathrm{EC}(C)$.

Let $a \in\{0,1\}^{n}$ by any input. If $\operatorname{psens}(f, a)=0$, the claim holds. Hence we can assume, $\operatorname{psens}(f, a) \neq 0$. Let $T$ be the set of all target nodes in $C$. For every $i \in \widetilde{\operatorname{psens}}(f, a)$, by Lemma 4.1, there exists continuous positive paths starting from $x_{i}$ to a gate $g \in T$.

For every $g \in T$, let $X_{g}$ be the set of all gates that lie in a continuous positive path from an $x_{i}$ to $g$ for some $i \in \widetilde{\operatorname{psens}}(f, a)$. Note that the subgraph induced by vertices in $X_{g}$ is connected and does not include $g$. We now obtain a connected DAG with psens $(f, a)$ leaves as follows. Let $D$ be a full binary tree (with edges directed from child to parent) with $|T|$ many leaves and hence $|T|-1$ internal nodes. For each $g \in T$ if it is a negation, we attach the gate feeding into $g$ as a leaf of the $D$ and if it is a root, we attach the root as a leaf of the $D$. We call the resulting DAG as $H$.

Let $X=\cup_{g \in T} X_{g}$. We now argue that psens $(f, a) \leq|X|+|T|$. Observe that the number of internal nodes in the DAG $H$ is $|X|+(|T|-1)$ where the first term is the gates in $X$ and the second term is the number of internal nodes of the tree $D$. Since graph induced on $X_{g}$ is connected for each $g$, this results in the DAG $H$ to be connected with psens $(f, a)$ many source nodes. As $H$ is connected, the number of leaves, which is $\operatorname{psens}(f, a)$, is at most the number of internal nodes +1 which is $|X|+|T|-1+1=|X|+|T|$.

We now give a bound on $|X|$ and $|T|$. Recall that since the target gates include negations and the root, $|T|=\operatorname{negs}(C)+1$. Since negs $(C)$ in any circuit $C$ is at most $E C(C)$ (Proposition 2.3), $|T| \leq \mathrm{EC}(C)+1$. Since all gates in $X$ output 1 as they belong to some continuous positive path in $C,|X| \leq \mathrm{EC}(C)$. This implies that, $\operatorname{psens}(f, a) \leq|X|+|T| \leq 2 \mathrm{EC}(C)+1 \leq 3 \mathrm{EC}(C)$ as $f$ is non-constant.

This implies that since psens $\left(\wedge_{n}\right)=n$, for $\mathrm{EC}\left(\wedge_{n}\right) \geq n / 3$ which is asymptotically tight by Proposition 2.2. We observe that even thought $\wedge_{n}$ is symmetric, the result of Suzuki et al. [SUZ13] on the energy lower bound on threshold circuits computing symmetric functions (which applies to Boolean circuits too), only yields a trivial lower bound (see Table 1). We remark that both these bounds does not give any non-trivial energy lower bound for $f=\vee_{n}$. Note that Theorem 1.2 uses the fact that the circuits used have fan-in 2. If fan-in of the circuit $C$ is $c$, then replacing each gate by a tree of $c-1$ gates of fan-in 2, by a similar argument as before, $\mathrm{EC}(C) \geq \operatorname{psens}(f) /(c+1)$. 


\subsection{Energy Lower Bounds from Karchmer-Wigderson Games}

Proposition 2.4 says that the monotone circuits are not energy efficient for computing monotone functions. In this section, we explore the limits on how energy efficient can non-monotone circuits be in computing monotone functions by showing the following.

Theorem 1.3. Let $f:\{0,1\}^{n} \rightarrow\{0,1\}$ be a monotone function. Then $\mathrm{EC}(f)=\Omega\left(\mathrm{KW}^{+}(f)\right)$.

Our approach is to use Lemma 4.1 and utilize the existence of continuous positive paths to design a $\mathrm{KW}^{+}$protocol of cost $\mathrm{EC}(C) \log ($ fan-in $(C))$ in Lemma 4.2 which immediately proves the above theorem. For the perfect matching function $f_{P M}$ on a graph of $n$ edges since $\mathrm{KW}^{+}\left(f_{P M}\right)=$ $\Omega(\sqrt{n})$ [RW92], this implies that any circuit $C$ with constant fan-in computing $f_{P M}$ require an energy of $\Omega(\sqrt{n})$.

Recall that for $x \in f^{-1}(1)$ and $y \in f^{-1}(0), S_{f}^{+}(x, y) \stackrel{\text { def }}{=}\left\{i \mid x_{i}=1, y_{i}=0, i \in[n]\right\}$. Also, we call the set of all negation gates, along with the root gate of $C$ as the target gates of $C$.

Lemma 4.2. For a non-constant monotone Boolean function $f$, let Alice and Bob hold inputs $a \in f^{-1}(1)$ and $b \in f^{-1}(0)$ respectively. Let $C$ be any circuit computing $f$, and every gate in the circuit is either $a \wedge, \vee$ with fan-in of at most $c$ or a negation gate. Then, $\mathrm{KW}^{+}(f) \leq \mathrm{EC}(C) \log c$.

Proof. We argue that, without loss of generality it can be assumed that $\widetilde{\text { psens }}(f, a)=\left\{i \mid a_{i}=1\right\}$. Alice finds an $a^{\prime} \prec a$ with $f\left(a^{\prime}\right)=f(a)=1$ such that for any $a^{\prime \prime} \prec a^{\prime}, f\left(a^{\prime \prime}\right)=0$. Observe that $a^{\prime} \neq$ $0^{n}$ for otherwise, $f\left(0^{n}\right)=1$ and since $f$ is monotone, $f$ must be a constant which is a contradiction. By construction, every bit in $a^{\prime}$ which is 1 is sensitive. Since $a^{\prime} \prec a, S_{f}^{+}\left(a^{\prime}, b\right) \subseteq S_{f}^{+}(a, b)$, thereby it suffices to find an index in $S_{f}^{+}\left(a^{\prime}, b\right)$.

We now describe the protocol. Let $a \in f^{-1}(1)$ such that $\widetilde{\text { psens }}(f, a)=\left\{i \mid a_{i}=1\right\}$. Before the protocol begins, Alice does the following pre-computation. Let $\mathcal{P}$ be the collection of positive paths one each for every $i \in \widetilde{\operatorname{psens}}(f, a)$, which exists as per Lemma 4.1. Alice computes $\mathcal{P}=$ $\bigcup_{g \in T} \mathcal{P}_{g}$ where $\mathcal{P}_{g}$ is the collection of all continuous positive paths ending at the target gate $g$. This ends the pre-processing.

Now Alice and Bob fixes an ordering of the target gates. For each target gate $g \in T$ in the order, the following procedure is repeated. For each continuous positive path $p \in \mathcal{P}$, ending at $g$, Alice sends the address of the previous gate in the path $p$ (using $\log c$ bits) until they trace back to an input index $i$. Now, Bob checks if $b_{i}=0$, and if so, we have found $i \in S_{f}^{+}(a, b)$, else, they attempt on the next $p \in \mathcal{P}_{g}$.

We argue about the correctness of the protocol. Notice that the above protocol searches through all $i \in \widehat{\operatorname{psens}}(f, a)$ by traversing through all $\mathcal{P}_{g}$, for $g \in T$. Since $\widetilde{\operatorname{psens}}(f, a)=\left\{i \mid a_{i}=1\right\}$ and $S_{f}^{+}(a, b) \subseteq \widetilde{\operatorname{psens}}(f, a)$ the protocol correctly computes $i$ such that $a_{i}=1$ and $b_{i}=0$. We now analyze the cost of the protocol. Observe that whenever Alice and Bob encounters a new path in $\mathcal{P}$ with parts of the path already traversed, they can move along the edges without exchange of any address. Hence, a gate in a path belonging to $\mathcal{P}$ gets visited at most once. Since the protocol visits only those gates that output 1 at most once on the input $a$, we have a protocol with communication cost $\leq \mathrm{EC}(C, a) \times \log (c) \leq \mathrm{EC}(C) \log c$.

\subsection{Energy Lower Bounds for Depth Three Circuits}

We now consider lower bounds on the energy complexity of constant depth (unbounded fan-in) circuits computing the parity function on $n$ bits. Observe that the energy complexity of circuits 
of unbounded fan-in can be very small compared to bounded fan-in circuits ${ }^{6}$. Hence the results proved in the bounded fan-in setting ( Theorem 1.2) does not directly apply.

For any Boolean function $f$, the trivial depth 2 circuit of unbounded fan-in computing $f$ has an energy $n+2$ and it can be shown that any depth two Boolean circuit computing the parity on $n$ bits require an energy of $n+1$.

Proposition 4.3. Let $C$ be any depth 2 circuit computing $\oplus_{n}$. Then $\mathrm{EC}(C) \geq n+1$.

Proof. Since $C$ computes $\oplus_{n}$, no variable or its negation can feed into the root gate, and every variable or its negation must feed into all gates at depth 2.

We now argue that at least $n-1$ variables must be negated in $C$. Suppose not, then there must be two variables, say $x_{i}$ and $x_{j}$, that feeds into all the gates in depth 2 unnegated. Setting $x_{i}=0$, all the $\wedge$ gates at depth 2 must evaluate to 0 . Similarly, setting $x_{j}=1$, causes all $\vee$ gates in depth 2 to evaluate to 1 . Hence the circuit $C$ evaluates to a fixed value irrespective of the remaining $n-2$ inputs unset which is a contradiction. Thus we conclude that at least $n-1$ variables must be negated. Consider an input $x$ that is 0 on these $n-1$ negated variables and 1 on the remaining variable. On this input, all the negation gates, the $\wedge$ gate which they feed into and the root gate evaluates to 1 . Hence $\mathrm{EC}(C) \geq \operatorname{EC}(C, x) \geq n-1+1+1=n+1$.

Santha and Wilson [SW93] showed that for any unbounded fan-in circuit $C$ of depth $d$ computing $\oplus_{n}, \operatorname{negs}(C) \geq d(\lceil n / 2\rceil)^{1 / d}-d$. Since energy complexity of a circuit $C$ is at least the number of negation gates in $C$ (Proposition 2.3), this implies that $\mathrm{EC}(C) \geq d(\lceil n / 2\rceil)^{1 / d}-d$ for any such circuit $C$ computing $\oplus_{n}$.

While we are unable to prove strong lower bounds for circuits of depth $d$ for an arbitrary constant $d$, we show that any depth $d=3$ unbounded fan-in circuit computing the parity function requires large energy. We achieve this by appealing to the known lower bounds on size of any constant depth circuit computing $\oplus_{n}$. Razborov showed that any circuit $C$ of depth $d$ of unbounded fan-in computing parity on $n$ bits must be of size at least $2^{\Omega\left(n^{1 / 4 d}\right)}$ [Raz87]. Using this result we show an energy lower bound of $\Omega(n)$ for any depth 3 circuit computing $\oplus_{n}$.

Theorem 1.4. Let $C$ be any unbounded fan-in circuit of depth 3 computing the parity function on $n$ variables. Then, $\mathrm{EC}(C)=\Omega(n)$.

Proof. We call the root gate of the circuit as the "top" level and the two level immediately below as the "middle" and "bottom" levels respectively. Note that negations can appear anywhere in the circuit and does not count towards the level. Assume without loss of generality that the circuit $C$ does not have any redundant gates.

Let there be $i$ negated input variables and without loss of generality assume $i<n$. We set these variables to 0 and let $C^{\prime}$ be the resulting circuit obtained. Let $g_{1}, g_{2}, \ldots, g_{k}$ be the $k$ gates in the bottom layer that feed to the layers above via negation gates. We set input variables to these $k$ gates such that the output of the negations are fixed in the following way: for the gate $g_{i}$, consider any input variable, say $x_{j}$, that feed into $g_{i}$ and set it to 0 if $g_{i}$ is $\wedge$ gate and 1 if $g_{i}$ is $\vee$. We also remove the gates that have become a constant and hardwire their output to get the resulting circuit $C^{\prime}$. Hence, all the gates at the bottom level are not fed negated to the level above.

\footnotetext{
${ }^{6}$ For instance, $\wedge_{n}$ has a fan-in $n$ circuit of depth 1 and energy 1 computing it while, by Theorem 1.2, energy of any bounded fan-in circuit computing the same function is $\Omega(n)$.
} 
In this process, we have eliminated the $k$ negations leaving us with the circuit $C^{\prime \prime}$ where all the gates at bottom and middle layer computes some monotone function on the remaining $m=$ $n-(i+j)$ for some $j \leq k$ variables. Since the resulting circuit must compute parity on $m$ variables, by [Raz87], size $\left(C^{\prime \prime}\right) \geq 2^{\Omega\left(m^{1 / 12}\right)}$. Since $C^{\prime \prime}$ is of depth 3 , the number of bottom and middle gates in $C^{\prime}$ must also be at least $2^{\Omega\left(m^{1 / 12}\right)}$. As the gates in the bottom and middle level computes monotone function, setting all the variables to 1 in $C^{\prime \prime}$ forces all of them must output 1 (Here we use the fact that the redundant gates are eliminated in $C$ ). Hence in $C$, there is a setting of input such that at least $i+k \geq i+j=n-m$ gates contributes an energy of 1 (since either the input to the negation or the negation gate itself will be 1$)$ and $2^{\Omega\left(m^{1 / 12}\right)}$ gates in $C$ that evaluate to 1 . Hence, $\mathrm{EC}(C) \geq n-m+2^{\Omega\left(m^{1 / 12}\right)}$. Let $c$ be the smallest integer such that for all $t \geq c, 2^{\Omega\left(t^{1 / 12}\right)}$ is at least $t$. There can be two cases:

1. If $m \geq c$, then $\operatorname{EC}(C) \geq n-m+2^{\Omega\left(m^{1 / 12}\right)} \geq n$,

2. Otherwise, $m<c$ and $\mathrm{EC}(C) \geq n-m+2^{\Omega\left(m^{1 / 12}\right)} \geq n-c+1=\Omega(n)$ as $c$ is independent of $n$.

Hence $\mathrm{EC}(C)=\Omega(n)$.

\section{Energy Complexity of Boolean Formulas}

For a formula $F$, let $\mathrm{L}(F)$ denote the number of leaves in the formula $F$. For any formula $F$, we have $\mathrm{EC}^{\mathrm{F}}(F) \leq 2 \mathrm{~L}(F)-1$ as $F$ has $\mathrm{L}(F)-1$ internal nodes and can have at most $\mathrm{L}(F)$ negation gates as leaves (in the worst case). Unlike circuits, any sub-function computed in a formula cannot be reused which can potentially lead to many gates that output a 1 on some input. For this reason, one would expect that it is unlikely for Boolean formulas to be energy efficient. As a warm up, we first implement the above argument for structured Boolean formulas where we prove strong lower bounds of $\Omega(L(F))$ (Section 5.1) and discuss its limitations. Then, using a different approach, we show a weaker lower bound of $\Omega(\sqrt{\mathrm{L}(F)}-\operatorname{Depth}(F))$ (Section 5.2) for arbitrary Boolean formulas.

\subsection{A Warm up}

We consider the following approach to prove a lower bound on energy complexity of a formula $F$ by exhibiting an input on which many gates are guaranteed to output a 1 . Suppose $t$ be the number of gates in a formula which have both its inputs as variables. We call such gates as non-skew gates. Now, set the $n$ variables to 0 or 1 uniformly at random. Then, each of the $t$ gates evaluate to a 1 with probability at least $1 / 4$. Hence, on expectation, there are at least $t / 4$ such gates evaluating to 1. This implies the existence of an input on which $\Omega(t)$ gates fire which gives the following proposition.

Proposition 5.1. For a formula $F$, let $t$ be the number of non-skew gates in F. If $t=\Omega(\mathrm{L}(F))$, then $\mathrm{EC}^{\mathrm{F}}(F)=\Theta(\mathrm{L}(F))$.

However, this argument fails ${ }^{7}$ for formulas where the gates are skew (i.e. exactly one of the input to the gate is a variable) since randomly setting the input does not necessarily guarantee

\footnotetext{
${ }^{7}$ In the conference version of this paper [DOS18], it was erroneously claimed that Proposition 5.1 holds for all Boolean formulas (that is, irrespective of $t$ ).
} 
a constant probability for the skew part to output a 1 (for example, consider formulas whose underlying graph is the fully right skewed tree). Hence, this approach does not give a lower bound for $\mathrm{EC}^{\mathrm{F}}(F)$ for an arbitrary formula $F$.

Nevertheless, there can be special formulas for which we can prove the lower bound of $\Omega(\mathrm{L}(F))$. For instance, consider the read-once formulas with negations at leaf. Similar to the argument of energy of monotone circuits (Proposition 2.4), the following can be concluded about them (irrespective of the formula structure).

Proposition 5.2. For any read-once formula $F$ with negations at the leaf, $\mathrm{EC}^{\mathrm{F}}(F) \geq \mathrm{L}(F)-1$.

\subsection{Bounds on Energy Complexity for Boolean Formulas}

In this section, we take a different approach and show the following lower bound on the energy complexity of any Boolean formula.

Theorem 1.5. For a Boolean function $f$, computed by a formula $F$,

$$
\mathrm{EC}^{\mathrm{F}}(F)=\Omega(\sqrt{\mathrm{L}(F)}-\operatorname{Depth}(F)) .
$$

Though the above result applies for any Boolean formula, it does not give any non-trivial lower bound for formulas that have large depths due to presence of long path of skew gates.

We now describe our approach. The main idea is to use a structural decomposition result for Boolean formulas due to Guo and Komargodski [GK17] (see also Tal [Tal14]). They showed that any formula $F$ can be transformed to another "structured formula" $F^{\prime}$ without blowing up the size. More precisely,

Theorem 5.3 (Theorem 3.1 of [GK17]). Let a Boolean function $f$ be computed by a Boolean formula $F$ with negs $(F) \geq 1$. Then, there exists $T \leq 5 \operatorname{negs}(F)-2$ monotone functions $g_{1} \ldots, g_{T}$ where each $g_{i}$ is computed by a monotone formula $G_{i}$ and a function $h:\{0,1\}^{T} \rightarrow\{0,1\}$ computed by a read-once formula $H$ such that $f(x)=h\left(g_{1}, \ldots, g_{T}\right)$ computed by the formula $F^{\prime} \stackrel{\text { def }}{=} H\left(G_{1}, \ldots, G_{T}\right)$ satisfy $L\left(F^{\prime}\right) \leq 2 \mathrm{~L}(F)$.

Before proceeding, we illustrate this result for a simple case (which we use later) when the formula $F$ has exactly one negation gate which it not at the root.

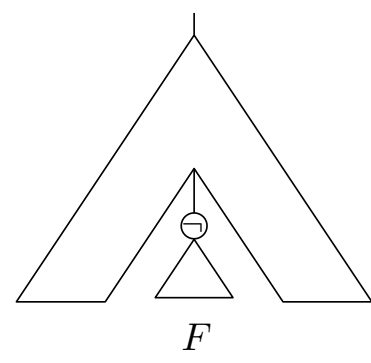

(a) Formula $F$

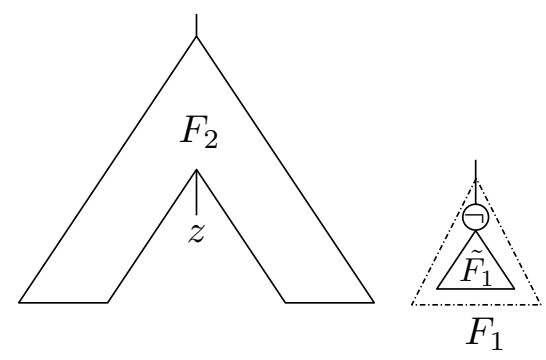

(b) Sub formulas $F_{1}$ and $F_{2}$ of $F$

Figure 3: Theorem 3.1 of [GK17] for formula $F$ with negs $(F)=1$

Fig. 3(a) shows the formula $F$ with exactly one negation gate and Fig. 3(b) shows two subformulas $F_{1}$ and $F_{2}$ with $F_{2}$ and $\tilde{F}_{1}$ being monotone. 


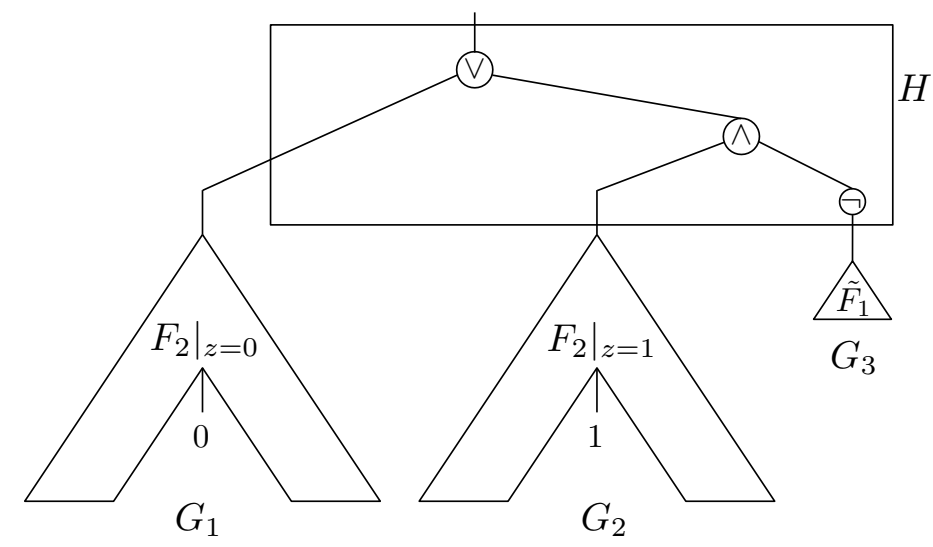

Figure 4: Structured formula $F^{\prime}$ obtained when Theorem 3.1 of [GK17] is applied to $F$ in Fig. 3

The resulting formula obtained upon applying Theorem 5.3 is $F^{\prime}=\left.F_{2}\right|_{z=0} \vee\left(\left.F_{2}\right|_{z=1} \wedge \tilde{F}_{1}\right)$ as shown in Fig. 4. The monotone formulas $G_{1}, G_{2}$ and $G_{3}$ are $\left.F_{2}\right|_{z=0},\left.F_{2}\right|_{z=1}$ and $\tilde{F}_{1}$ respectively. The read-once formula $H$ is $H\left(y_{1}, y_{2}, y_{3}\right)=y_{1} \vee\left(y_{2} \wedge \neg y_{3}\right)$. Also, $\mathrm{L}\left(F^{\prime}\right) \leq 2 \mathrm{~L}(F)$.

We now describe our proof strategy: firstly, we analyze the energy of the formula $F^{\prime}$ obtained in Theorem 5.3 and show (in Lemma 5.5) that $\mathrm{EC}^{\mathrm{F}}\left(F^{\prime}\right)$ is upper bounded asymptotically by $O(\operatorname{negs}(F) \times$ $\left(\mathrm{EC}^{\mathrm{F}}(F)+\operatorname{Depth}(F)\right)$. This implies that the decomposition in Theorem 5.3 is not only size efficient but also energy efficient. The specific structure of the formula from Theorem 5.3 implies that $\mathrm{EC}^{\mathrm{F}}\left(F^{\prime}\right)$ is lower bounded by $\Omega(\mathrm{L}(F)-\operatorname{negs}(F)$ ) (Lemma 5.6). Finally, comparing the upper and lower bound for $\mathrm{EC}^{\mathrm{F}}\left(F^{\prime}\right)$ gives a lower bound on $\mathrm{EC}^{\mathrm{F}}(F)$ in terms of $\mathrm{L}(F)$, $\operatorname{Depth}(F)$ and negs $(F)$ using which we prove Theorem 1.5. Before proceeding, we need the following observation.

Proposition 5.4. Let $F$ be any formula and $g$ be any gate of $F$ other than the root. Let $D$ be a formula obtained by replacing the subtree at gate $g$ by a variable $z$. Then for any $b \in\{0,1\}, \operatorname{EC}\left(\left.D\right|_{z=b}\right) \leq$ $\mathrm{EC}^{\mathrm{F}}(F)+\operatorname{Depth}(F)$.

Proof. Fix a $b \in\{0,1\}$ and let $a$ be an input on which $\left.D\right|_{z=b}$ achieves the maximum energy. Consider the evaluation of gates in $F$ on this input $a$. If we ignore the gates in the subtree rooted at $g$ in $F$, as $F$ is a formula, the evaluation of gates on the input $a$ for $F$ and $\left.D\right|_{z=b}$ can differ only on those gates that lie in the path from $g$ to the root. Hence,

$$
\operatorname{EC}^{\mathrm{F}}(F) \geq \operatorname{EC}^{\mathrm{F}}(F, a) \geq \operatorname{EC}\left(\left.D\right|_{z=b}, a\right)-\operatorname{Depth}(F)=\mathrm{EC}\left(\left.D\right|_{z=b}\right)-\operatorname{Depth}(F)
$$

which completes the proof.

Lemma 5.5 (Upper Bound for $\operatorname{EC}^{\mathrm{F}}\left(F^{\prime}\right)$ ). Let $f:\{0,1\}^{n} \rightarrow\{0,1\}$ be computed by a Boolean formula $F$ with negs $(F) \geq 1$. Then, the formula $F^{\prime}$ computing $f$ obtained by applying the decomposition of Theorem 5.3 to $F$ satisfies,

$$
\mathrm{EC}^{\mathrm{F}}\left(F^{\prime}\right) \leq(5 \operatorname{negs}(F)-2)\left(\mathrm{EC}^{\mathrm{F}}(F)+\operatorname{Depth}(F)+1\right)
$$

Proof. We proceed by tracing the construction in Theorem 3.1 of [GK17] (Theorem 5.3) where we bound the energy of the resulting formula thereby proving the result.

[By strong induction on negs $(F)$ ] For the base case with negs $(F)=1$, let $F_{1}$ be the minimal formula containing all negations of $F$. If $F_{1}=F$, then the root gate of $F$ must be a NOT gate and $F^{\prime}=F$ 
satisfies Eq. (1). Otherwise, let $F_{2}$ be the formula obtained by replacing $F_{1}$ in $F$ by a new variable $z$. As $F_{1}$ has the only negation gate of $F, F_{2}$ is monotone implying $F_{2}=\left.F_{2}\right|_{z=0} \vee\left(\left.F_{2}\right|_{z=1} \wedge z\right)$. Also there exists a formula $\tilde{F}_{1}$ such that $F_{1}=\neg \widetilde{F}_{1}$ (see Fig. 3). Now the formula $F^{\prime}=\left.F_{2}\right|_{z=0} \vee\left(\left.F_{2}\right|_{z=1} \wedge\right.$ $\left.\neg \tilde{F}_{1}\right)$ computes the same function as $F$. Since $\operatorname{EC}^{\mathrm{F}}\left(F^{\prime}\right)$ is upper bounded by the energy of the individual formulas and the connecting gates,

$$
\begin{aligned}
\mathrm{EC}^{\mathrm{F}}\left(F^{\prime}\right) & \leq \mathrm{EC}^{\mathrm{F}}\left(\left.F_{2}\right|_{z=0}\right)+\mathrm{EC}^{\mathrm{F}}\left(\left.F_{2}\right|_{z=1}\right)+\mathrm{EC}\left(\tilde{F}_{1}\right)+3 & & \\
& \leq 2 \mathrm{EC}^{\mathrm{F}}(F)+2 \operatorname{Depth}(F)+\mathrm{EC}\left(\tilde{F}_{1}\right)+3 & & {[\text { Proposition 5.4] }} \\
& \leq 3\left(\mathrm{EC}^{\mathrm{F}}(F)+\operatorname{Depth}(F)+1\right) & & {\left[\widetilde{F}_{1} \text { is a subformula of } F\right] }
\end{aligned}
$$

For the inductive case, let $F$ be any Boolean formula with $t=\operatorname{negs}(F)>1$ and the result holds for all formulas with negations less than $t$. Let $F_{1}$ be the smallest subformula of $F$ that contains all the negations of $F$. There can be two cases.

Case 1. $F_{1}$ is same as $F$ : In this case, we show that there is an $F^{\prime}$ computing the same function as $F$ with $\operatorname{EC}^{\mathrm{F}}\left(F^{\prime}\right) \leq(5$ negs $(F)-4)\left(\mathrm{EC}^{\mathrm{F}}(F)+\operatorname{Depth}(F)+1\right)$ satisfying Eq. (1). Based on the root gate of $F$, there can be two subcases.

Suppose the root of $F$ is a NOT gate. Then, there exists a formula $E$ such that $F=\neg E$. Since negs $(E)=\operatorname{negs}(F)-1$, by induction, there exists an $E^{\prime}$ computing the same function as $E$ with $\operatorname{EC}^{\mathrm{F}}\left(E^{\prime}\right) \leq(5 \operatorname{negs}(E)-2)\left(\operatorname{EC}^{\mathrm{F}}(E)+\operatorname{Depth}(E)+1\right)$. Now the formula $F^{\prime}=\neg E^{\prime}$ computes the same function as $F$. Estimating $\operatorname{EC}^{\mathrm{F}}\left(F^{\prime}\right)$, we have

$$
\begin{array}{rlrl}
\operatorname{EC}^{\mathrm{F}}\left(F^{\prime}\right) & \leq \operatorname{EC}^{\mathrm{F}}\left(E^{\prime}\right)+1 & \\
& \leq(5 \operatorname{negs}(E)-2)\left(\operatorname{EC}^{\mathrm{F}}(E)+\operatorname{Depth}(E)+1\right)+1 & & {[\text { Induction }]} \\
& \leq(5(\operatorname{negs}(F)-1)-2)\left(\mathrm{EC}^{\mathrm{F}}(E)+\operatorname{Depth}(F)\right)+1 & & {[\operatorname{Depth}(E)=\operatorname{Depth}(F)-1]} \\
& \leq(5 \operatorname{negs}(F)-4)\left(\operatorname{EC}^{\mathrm{F}}(F)+\operatorname{Depth}(F)+1\right) & & {\left[\operatorname{EC}^{\mathrm{F}}(E) \leq \operatorname{EC}^{\mathrm{F}}(F)+1\right]}
\end{array}
$$

Suppose the root of $F$ is AND/OR. Without loss of generality, let the root be OR gate. A similar argument holds for the case of AND gate. Then, let $F=E_{\ell} \vee E_{r}$. where $E_{\ell}, E_{r}$ are the left and right subtrees of the root, respectively. Since $E_{\ell}$ and $E_{r}$ are subformulas of $F$, observe that $\mathrm{EC}^{\mathrm{F}}\left(E_{\ell}\right) \leq \mathrm{EC}^{\mathrm{F}}(F)$ and $\mathrm{EC}^{\mathrm{F}}\left(E_{r}\right) \leq \mathrm{EC}^{\mathrm{F}}(F)$. Since $F_{1}=F$, it must be that negs $\left(E_{\ell}\right) \geq 1$ and negs $\left(E_{r}\right) \geq 1$. Hence, by induction, there exists formulas $E_{\ell}^{\prime}$ and $E_{r}^{\prime}$ computing the same function as $E_{\ell}$ and $E_{r}$, respectively. Consider the formula $F^{\prime}=E_{\ell}^{\prime} \vee E_{r}^{\prime}$. We now show that $F^{\prime}$ satisfies the required energy bound.

$$
\begin{aligned}
\operatorname{EC}^{\mathrm{F}}\left(F^{\prime}\right) \leq & \operatorname{EC}^{\mathrm{F}}\left(E_{\ell}^{\prime}\right)+\operatorname{EC}^{\mathrm{F}}\left(E_{r}^{\prime}\right)+1 \\
\leq & \left(5 \operatorname{negs}\left(E_{\ell}\right)-2\right)\left(\operatorname{EC}^{\mathrm{F}}\left(E_{\ell}\right)+\operatorname{Depth}\left(E_{\ell}\right)+1\right) \\
& +\left(5 \operatorname{negs}\left(E_{r}\right)-2\right)\left(\mathrm{EC}^{\mathrm{F}}\left(E_{r}\right)+\operatorname{Depth}\left(E_{r}\right)+1\right)+1 \quad[\text { Induction] } \\
\leq & \left(5 \operatorname{negs}\left(E_{\ell}\right)-2\right)\left(\mathrm{EC}^{\mathrm{F}}(F)+\operatorname{Depth}(F)\right) \\
& +\left(5 \operatorname{negs}\left(E_{r}\right)-2\right)\left(\mathrm{EC}^{\mathrm{F}}(F)+\operatorname{Depth}(F)\right)+1 \quad\left[\operatorname{Depth}\left(E_{\ell}\right), \operatorname{Depth}\left(E_{r}\right) \leq \operatorname{Depth}(F)-1\right] \\
\leq & (5 \operatorname{negs}(F)-4)\left(\operatorname{EC}^{\mathrm{F}}(F)+\operatorname{Depth}(F)+1\right)
\end{aligned}
$$


Case 2. $F_{1}$ is not same as $F$ : Let $F_{2}$ be the formula obtained by replacing $F_{1}$ in $F$ by a new variable $z$. Similar to the argument in the base case, $F^{\prime}=\left.F_{2}\right|_{z=0} \vee\left(\left.F_{2}\right|_{z=1} \wedge F_{1}\right)$ computes the same function as $F$. Since $F_{1}$ does not have a smaller subformula containing all its negations, we can apply Case 1 to $F_{1}$ to get a formula $F_{1}^{\prime}$ computing same function as $F_{1}$ with $\operatorname{EC}^{\mathrm{F}}\left(F_{1}^{\prime}\right) \leq$ $\left(5 \operatorname{negs}\left(F_{1}\right)-4\right)\left(\operatorname{EC}^{\mathrm{F}}\left(F_{1}\right)+\operatorname{Depth}\left(F_{1}\right)+1\right)$. Hence,

$$
\begin{array}{rlr}
\operatorname{EC}^{\mathrm{F}}\left(F^{\prime}\right) \leq & \mathrm{EC}^{\mathrm{F}}\left(\left.F_{2}\right|_{z=0}\right)+\mathrm{EC}^{\mathrm{F}}\left(\left.F_{2}\right|_{z=1}\right)+\mathrm{EC}^{\mathrm{F}}\left(F_{1}^{\prime}\right)+2 & \\
\leq & 2 \mathrm{EC}^{\mathrm{F}}(F)+2 \operatorname{Depth}(F)+\operatorname{EC}^{\mathrm{F}}\left(F_{1}^{\prime}\right)+2 & \\
\leq & \left(5 \operatorname{negs}\left(F_{1}\right)-4\right)\left(\mathrm{EC}^{\mathrm{F}}\left(F_{1}\right)+\operatorname{Depth}\left(F_{1}\right)+1\right) & \\
& +2\left(\operatorname{EC}^{\mathrm{F}}(F)+\operatorname{Depth}(F)+1\right) & \\
\leq & (5 \operatorname{negs}(F)-2)\left(\operatorname{EC}^{\mathrm{F}}(F)+\operatorname{Depth}(F)+1\right) & {\left[F_{1} \text { is a subformula of } F\right]}
\end{array}
$$

Lemma 5.6 (Lower Bound for $\operatorname{EC}^{\mathrm{F}}\left(F^{\prime}\right)$ ). Let $F$ be a formula and $F^{\prime}$ be the formula obtained by applying Theorem 5.3 to F. Then, $\mathrm{EC}^{\mathrm{F}}\left(F^{\prime}\right) \geq \mathrm{L}(F)-(5 \operatorname{negs}(F)-2)$.

Proof. By Theorem 5.3 the $F^{\prime}$ obtained is a composition of a read-once formula $H$ over monotone formulas $G_{1}, \ldots, G_{T}$ for $T \leq 5 \operatorname{negs}(F)-2$. In addition, by tracing the construction of $F^{\prime}$ in the proof of Theorem 5.3, it can be inferred that (1) all leaves of $F^{\prime}$ forms a part of some monotone formula $G_{i}$ and (2) every leaf in $F$ must appear at least once as a leaf of $F^{\prime}$. Now,

$$
\begin{array}{rlrl}
\operatorname{EC}^{\mathrm{F}}\left(F^{\prime}\right) & \geq \operatorname{EC}^{\mathrm{F}}\left(F^{\prime}, 1^{n}\right) & \\
& \geq \sum_{i=1}^{T} \operatorname{EC}^{\mathrm{F}}\left(G_{i}, 1^{n}\right) & \\
& \geq \sum_{i=1}^{T}\left(\mathrm{~L}\left(G_{i}\right)-1\right) & & {\left[G_{i} \text { s are monotone }\right]} \\
& \geq \mathrm{L}(F)-T & & {[\text { By Property }(1) \text { and }(2)]} \\
& \geq \mathrm{L}(F)-(5 \operatorname{negs}(F)-2) & &
\end{array}
$$

Theorem 1.5 holds directly from the following cumbersome but slightly stronger claim.

Claim 5.7. For any formula $F, \mathrm{EC}^{\mathrm{F}}(F)=\Omega\left(\sqrt{\mathrm{L}(F)+\operatorname{Depth}(F)^{2}+\operatorname{Depth}(F)}-\operatorname{Depth}(F)\right)$.

Proof. If negs $(F)=0$, then $F$ is monotone and $\mathrm{EC}^{\mathrm{F}}(F)=\operatorname{EC}^{\mathrm{F}}\left(F, 1^{n}\right)=\mathrm{L}(F)-1$. Otherwise, $\operatorname{negs}(F) \geq 1$ and applying Lemma 5.5 we have $\operatorname{EC}^{\mathrm{F}}\left(F^{\prime}\right) \leq(5 \operatorname{negs}(F)-2)\left(\operatorname{EC}^{\mathrm{F}}(F)+\operatorname{Depth}(F)+1\right)$ and by Lemma 5.6 the formula $F^{\prime}$ obtained satisfy, $\operatorname{EC}^{\mathrm{F}}\left(F^{\prime}\right) \geq \mathrm{L}(F)-(5 \operatorname{negs}(F)-2)$.

Combining the two bounds on $\operatorname{EC}^{\mathrm{F}}\left(F^{\prime}\right)$, we have $\mathrm{EC}^{\mathrm{F}}(F) \geq \frac{\mathrm{L}(F)}{5 \operatorname{negs}(F)-2}-\operatorname{Depth}(F)-2$. Along with Proposition 2.3, we have

$$
\operatorname{EC}^{\mathrm{F}}(F) \geq \max \left\{\frac{\mathrm{L}(F)}{5 \operatorname{negs}(F)-2}-\operatorname{Depth}(F)-2, \operatorname{negs}(F)\right\}
$$


Let $\alpha$ be the largest possible value such that $\frac{\mathrm{L}(F)}{5 \alpha-2}-\operatorname{Depth}(F)-2 \geq \alpha$. This gives a quadratic equation in $\alpha$ and it can be verified that the maximizing $\alpha$ is $\frac{\sqrt{(5 \operatorname{Depth}(F)+12)^{2}+20 \mathrm{~L}(F)}-(5 \operatorname{Depth}(F)+8)}{10}$.

If negs $(F)$ is at least $\alpha$, then $\operatorname{EC}^{\mathrm{F}}(F) \geq \alpha$. Otherwise, $\operatorname{EC}^{\mathrm{F}}(F)$ is lower bounded by $\frac{\mathrm{L}(F)}{5 \alpha-2}-$ $\operatorname{Depth}(F)-2$ which, by our choice, is at least $\alpha$. Hence in both cases,

$$
\mathrm{EC}^{\mathrm{F}}(F) \geq \alpha=\Omega\left(\sqrt{\mathrm{L}(F)+\operatorname{Depth}(F)^{2}+\operatorname{Depth}(F)}-\operatorname{Depth}(F)\right) .
$$

\section{Comparison of Lower Bound Techniques for Energy Complexity and a Recent Improvement}

So far, we have seen two techniques to show lower bound for energy complexity one in terms of positive sensitivity (Theorem 1.2) for any Boolean function and other in terms of cost of monotone Karchmer-Wigderson game (Theorem 1.3) for monotone Boolean functions. In this section, we give a comparison of lower bound techniques for energy complexity with regard to a recent improvement due to Sun et al. [SSWX19].

Theorem 1.1 says that every Boolean function of small decision tree depth has a small energy circuit computing it. In the context of proving lower bounds on energy complexity, a natural question to ask is whether a converse of Theorem 1.1 is true. That is, does a circuit of small energy have a small depth decision tree computing it. More precisely,

Question 6.1. Is it true that for all Boolean functions $f$, DT $(f) \leq \operatorname{poly}(\operatorname{EC}(f))$ ?

In this context, we give our approach to answer this question using a measure called maxentropy of a circuit introduced by Uchizawa et al. [UDM06]. For a circuit $C$, the max-entropy, denoted by $\mathrm{H}_{\max }(C)$ is the logarithm of the number of firing patters of the circuit $C$. As a part of main result, they showed that for any threshold circuit $C$ computing a Boolean function $f$, $\mathrm{H}_{\max }(C) \geq \mathrm{EC}_{\mathcal{T}}(f)-1$. Hence $\mathrm{H}_{\max }(C)$ can be seen as yet another measure of energy complexity for threshold circuits.

Since the same result does not directly extend to circuits over Boolean basis $\mathcal{B}$, we ask, in a spirit similar to the result of Uchizawa et al. [UDM06], if max-entropy is also a measure of energy for Boolean circuits. We show in Lemma 6.2 an analogous result for Boolean circuits that for any Boolean function $f$, and a circuit $C$ computing $f, \mathrm{H}_{\max }(C)=\Omega(\log \mathrm{DT}(f))$.

Lemma 6.2. For a Boolean function $f:\{0,1\}^{n} \rightarrow\{0,1\}$, let $C$ be any Boolean circuit computing $f$ having gates computing an arbitrary function of a finite arity. Then, DT $(f)$ is, asymptotically, at most the number of firing patterns of $C$. Hence $\mathrm{H}_{\max }(C)=\Omega(\log \mathrm{DT}(f))$.

Proof. Let the number of firing patterns of $C$ be $t$ and $\ell$ be the maximum arity of gates in $C$. We show that there exists a decision tree computing $f$ of depth $\ell \cdot t$. Since $\ell$ is a finite constant, $t \geq$ $\mathrm{DT}(f) / \ell=\Omega(\mathrm{DT}(f))$.

Proof is by strong induction on $n$. For $n=1, \mathrm{DT}(f) \leq 1$ and there must be at least one firing pattern for $C$. Hence DT $(f) \leq \ell \cdot t$. Suppose the claim holds for all Boolean functions on $<n$ variables. Let $f$ be an $n$ bit Boolean function computed by a circuit $C$ of size $s$ with gates of fan-in at 
most $\ell$. For the circuit $C$, let there be $t$ distinct firing patterns $p_{1}, p_{2}, \ldots, p_{t}$ where each $p_{i} \in\{0,1\}^{s}$. Let $C^{\prime}$ be the circuit obtained from $C$ by removing all the gates that have the same value in all the firing patterns. Observe that this transformation does not alter the number of firing patterns and let $p_{1}^{\prime}, p_{2}^{\prime}, \ldots, p_{t}^{\prime}$ be the firing patterns of $C^{\prime}$. Let $g$ be a gate in $C^{\prime}$ whose evaluation depends only on input variables. Let $f^{\prime}$ be the function $f$ after setting the queried variables to the values read. Also set the queried values in $C^{\prime}$ and evaluate the circuit (as far as possible) to get $C^{\prime \prime}$ which computes $f^{\prime}$. Since $f^{\prime}$ is on $\leq n-\ell$ variables, by induction, DT $\left(f^{\prime}\right) \leq \ell \times$ Number of firing patterns of $C^{\prime \prime}$.

Since the value of gate $g$ is fixed, $C^{\prime \prime}$ can have at most $t-1$ firing patterns (for otherwise, all the firing patterns have the same value for gate $g$ due to which $g$ would have been removed in $C^{\prime}$, a contradiction). Hence, DT $(f) \leq \mathrm{DT}\left(f^{\prime}\right)+\ell \leq \ell \times$ Number of firing patterns of $C^{\prime \prime}+\ell \leq$ $\ell \cdot(t-1)+\ell=\ell \cdot t$.

However, this result does not give a meaningful lower bound for energy complexity of $f$. To see this, a circuit with $s$ internal gates and energy $e$ can potentially have $\sum_{i=0}^{e}\left(\begin{array}{c}s \\ i\end{array}\right) \leq s^{e}+1$ firing patterns implying $\mathrm{H}_{\max }(C) \leq e \log s$. Now, Lemma 6.2 implies that $e=\Omega(\log \mathrm{DT}(f) / \log s)$. However, $\log \mathrm{DT}(f) / \log s=O(1)$ as $\mathrm{DT}(f)=O(s)$.

Lemma 6.2 can be seen as constructing a decision tree for $f$, given the firing patterns of a circuit computing $f$. Recently, Sun et al. [SSWX19] directly constructed a decision tree of depth $\operatorname{EC}(f)^{2}$, thereby implying the following.

Theorem 6.3 (Sun et al. [SSWX19]). For all Boolean functions $f, \mathrm{EC}(f) \geq \sqrt{\mathrm{DT}(f)}$.

This answers Question 6.1 in affirmative as DT $(f) \leq \mathrm{EC}(f)^{2}$. The original statement, Theorem 2 of Sun et al. [SSWX19], states that $\mathrm{EC}(f)=\Omega(\sqrt{\mathrm{DT}(f)})$. A careful analysis of their proof reveals that the asymptotic constant is actually 1. In this context, we give two instances where the result of Sun et al. [SSWX19] can be used to further improve our results from Section 4.1 and Section 4.2.

- We showed that $\mathrm{EC}\left(\wedge_{n}\right) \geq n / 3$ based on the measure positive sensitivity (Theorem 1.2). But it completely fails to give any non-trivial lower bound for $\operatorname{EC}\left(\vee_{n}\right)$ since psens $\left(\vee_{n}\right)=1$. Since $\mathrm{DT}\left(\mathrm{\vee}_{n}\right)=n$, by Theorem 6.3 this implies that $\mathrm{EC}\left(\vee_{n}\right) \geq \sqrt{n}$ (as observed by Sun et al. [SSWX19]).

- Consider the problem of STCONN which, given a directed graph $G$ on $\left(\begin{array}{l}n \\ 2\end{array}\right)$ edges and two vertices $s$ and $t$, asks if there is a path from $s$ to $t$ in $G$. It is known that $\mathrm{KW}^{+}$(STCONN) $=$ $\Omega\left(\log ^{2} n\right)$ [KW90]. Hence, Theorem 1.3 implies that EC(STCONN $)=\Omega\left(\log ^{2} n\right)$. It can be argued that DT(STCONN) $=\Omega\left(n^{2}\right)$. One way to see this is to observe that connectivity is a non-trivial monotone property of graphs and such properties have decision tree depth of $\Omega\left(n^{2}\right)$ [KSS84]. Hence, by Theorem 6.3 we get that EC(STCONN $) \geq \sqrt{\text { DT(STCONN })}=$ $\Omega(n)$ which vastly improves what could be inferred via our bound.

\section{Discussion and Questions}

Having studied $\mathrm{EC}(f)$ as a Boolean function parameter for different circuit models over the Boolean basis $\mathcal{B}$, following are some natural questions that are left unanswered.

- For unbounded fan-in circuits of depth 3, we showed an energy lower bound of $\Omega(n)$ for parity on $n$ bits (Theorem 1.4). The question here is to extend the same to arbitrary depth unbounded fan-in circuits. 
- For any Boolean formula $F$, we showed a lower bound for $\operatorname{EC}^{\mathrm{F}}(F)$ in terms of its size and depth (Theorem 1.5). Can we remove the dependence on depth thereby showing that for all Boolean functions $f, \mathrm{EC}^{\mathrm{F}}(f)=\Omega(\sqrt{\mathrm{L}(f)})$ ?

\section{Acknowledgments}

The authors would like to thank the anonymous reviewers for their constructive comments.

\section{References}

$\left[\mathrm{ABN}^{+}{ }^{14}\right]$ Antonios Antoniadis, Neal Barcelo, Michael Nugent, Kirk Pruhs, and Michele Scquizzato. Energy-efficient circuit design. In Innovations in Theoretical Computer Science, ITCS'14, Princeton, NJ, USA, January 12-14, 2014, pages 303-312, 2014.

[BNPS15] Neal Barcelo, Michael Nugent, Kirk Pruhs, and Michele Scquizzato. Almost all functions require exponential energy. In Mathematical Foundations of Computer Science 2015 - 40th International Symposium, MFCS 2015, Milan, Italy, August 24-28, 2015, Proceedings, Part II, pages 90-101, 2015.

[CDR86] Stephen Cook, Cynthia Dwork, and Rdiger Reischuk. Upper and lower time bounds for parallel random access machines without simultaneous writes. SIAM J. Comput., 15(1):87-97, 1986.

[DOS18] Krishnamoorthy Dinesh, Samir Otiv, and Jayalal Sarma. New bounds for energy complexity of Boolean functions. In Computing and Combinatorics - 24th International Conference, COCOON 2018, Qing Dao, China, July 2-4, 2018, Proceedings, pages 738-750, 2018.

[DS18] Krishnamoorthy Dinesh and Jayalal Sarma. Alternation, sparsity and sensitivity: Combinatorial bounds and exponential gaps. In Algorithms and Discrete Applied Mathematics - 4th International Conference, CALDAM 2018, Guwahati, India, February 15-17, 2018, Proceedings, pages 260-273, 2018.

[GK17] Siyao Guo and Ilan Komargodski. Negation-limited formulas. Theoretical Computer Science, 660:75-85, 2017. A preliminary version appeared in RANDOM 2015.

[Juk12] Stasys Jukna. Boolean function complexity : Advances and Frontiers. Algorithms and combinatorics. Springer, Berlin, Heidelberg, 2012.

[Kas92] Kasim-zade, O.M. On a measure of active circuits of functional elements. In Mathematical problems in cybernetics "Nauka", volume No. 4 (Russian), pages 218-228, 1992.

[Kis82] Gloria Kissin. Measuring energy consumption in VLSI circuits: a foundation. In Proceedings of the 14th Annual ACM Symposium on Theory of Computing, May 5-7, 1982, San Francisco, California, USA, pages 99-104, 1982.

[KN06] Eyal Kushilevitz and Noam Nisan. Communication complexity. Cambridge University Press, 2nd edition, 2006. 
[KSS84] Jeff Kahn, Michael E. Saks, and Dean Sturtevant. A topological approach to evasiveness. Combinatorica, 4(4):297-306, 1984.

[KW90] Mauricio Karchmer and Avi Wigderson. Monotone circuits for connectivity require super-logarithmic depth. SIAM J. Discrete Math., 3(2):255-265, 1990.

[LS15] S. A. Lozhkin and M. S. Shupletsov. Switching activity of Boolean circuits and synthesis of Boolean circuits with asymptotically optimal complexity and linear switching activity. Lobacherskii Journal of Mathematics, 36(4):450-460, 2015.

[Mar58] A. A. Markov. On the inversion complexity of a system of functions. J. ACM, 5(4):331334, October 1958.

[Raz87] Razborov A.A. Lower bounds on the size of constant-depth networks over a complete basis with logical addition. Mathematicheskie Zametki, 41(4):598-607, 1987. English translation in Mathematical Notes of the Academy of Sci. of the USSR, 41(4):333-338, 1987.

[RW92] Ran Raz and Avi Wigderson. Monotone circuits for matching require linear depth. J. ACM, 39(3):736-744, 1992. A preliminary version appeared in STOC 1990.

[SSWX19] Xiaoming Sun, Yuan Sun, Kewen Wu, and Zhiyu Xia. On the relationship between energy complexity and other Boolean function measures. In Computing and Combinatorics - 25th International Conference, COCOON 2019, Xi'an, China, July 29-31, 2019, Proceedings, pages 516-528, 2019.

[SUZ13] Akira Suzuki, Kei Uchizawa, and Xiao Zhou. Energy and fan-in of logic circuits computing symmetric Boolean functions. Theor. Comput. Sci., 505:74-80, 2013.

[SW93] Miklos Santha and Christopher Wilson. Limiting negations in constant depth circuits. SIAM J. Comput., 22(2):294-302, April 1993.

[Tal14] Avishay Tal. Shrinkage of De Morgan formulae by spectral techniques. In 55th IEEE Annual Symposium on Foundations of Computer Science, FOCS 2014, Philadelphia, PA, USA, October 18-21, 2014, pages 551-560, 2014.

[UDM06] Kei Uchizawa, Rodney J. Douglas, and Wolfgang Maass. On the computational power of threshold circuits with sparse activity. Neural Computation, 18(12):2994-3008, 2006.

[UNT10] Kei Uchizawa, Takao Nishizeki, and Eiji Takimoto. Energy and depth of threshold circuits. Theor. Comput. Sci., 411(44-46):3938-3946, 2010. A preliminary version appeared in FCT 2009.

[UT08] Kei Uchizawa and Eiji Takimoto. Exponential lower bounds on the size of constantdepth threshold circuits with small energy complexity. Theor. Comput. Sci., 407(13):474-487, 2008. A preliminary version appeared in CCC 2007.

[UTN11] Kei Uchizawa, Eiji Takimoto, and Takao Nishizeki. Size-energy tradeoffs for unate circuits computing symmetric Boolean functions. Theor. Comput. Sci., 412(8-10):773782, 2011. 
[Vai62] M. N. Vaintsvaig. On the power of networks of functional elements. In Soviet Physics Doklady, volume 6, page 545, 1962. 